\title{
A Systematic Review of Tissue Engineering Scaffold in Tendon Bone Healing in vivo
}

\begin{abstract}
Zimu Mao ${ }^{1,2 \dagger}$, Baoshi Fan ${ }^{1,2,3 \dagger}$, Xinjie Wang 1,2†, Ximeng Huang 1,2, Jian Guan 1,2, Zewen Sun ${ }^{4,5}$, Bingbing $X u^{1,2}$, Meng Yang ${ }^{1,2,3}$, Zeyi Chen ${ }^{1,2}$, Dong Jiang ${ }^{1,2 *}$ and Jiakuo $\mathrm{Yu}^{1,2 *}$

${ }^{1}$ Sports Medicine Department, Beijing Key Laboratory of Sports Injuries, Peking University Third Hospital, Beijing, China, ${ }^{2}$ Institute of Sports Medicine of Peking University, Beijing, China, ${ }^{3}$ School of Clinical Medicine, Weifang Medical University, Weifang, China, ${ }^{4}$ Qingdao University, Qingdao, China, ${ }^{5}$ Department of Sports Medicine, The Affiliated Hospital of Qingdao University, Qingdao, China
\end{abstract}

Background: Tendon-bone healing is an important factor in determining the success of ligament reconstruction. With the development of biomaterials science, the tissue engineering scaffold plays an extremely important role in tendon-bone healing and bone tissue engineering.

Edited by:

Bin Li,

Soochow University, China

Reviewed by:

Hong-Bin Lu,

Central South University, China

Xin Zhao,

Hong Kong Polytechnic University,

Hong Kong

*Correspondence:

Jiakuo Yu

yujiakuo@126.com

Dong Jiang

bysyjiangdong@126.com

tThese authors have contributed equally to this work

Specialty section:

This article was submitted to Tissue Engineering and Regenerative Medicine,

a section of the journal Frontiers in Bioengineering and Biotechnology

Received: 26 October 2020 Accepted: 03 February 2021

Published: 15 March 2021

Citation:

Mao Z, Fan B, Wang X, Huang $X$ Guan J, Sun Z, Xu B, Yang M, Chen Z, Jiang $D$ and $Y u J(2021)$ A Systematic Review of Tissue Engineering Scaffold in Tendon Bone Healing in vivo. Front. Bioeng. Biotechnol. 9:621483.

doi: $10.3389 /$ fbioe. 2021.621483
Materials and Methods: Electronic databases (PubMed, Embase, and the Web of Science) were systematically searched for relevant and qualitative studies published from 1 January 1990 to 31 December 2019. Only original articles that met eligibility criteria and healing in vivo were selected for analysis.

Results: The search strategy identified 506 articles, and 27 studies were included for full review including two human trials and 25 animal studies. Fifteen studies only used biomaterials like PLGA, collage, PCL, PLA, and PET as scaffolds to repair the tendon-bone defect, on this basis, the rest of the 11 studies using biological interventions like cells or cell factors to enhance the healing. The adverse events hardly ever occurred, and the tendon bone healing with tissue engineering scaffold was effective and superior, which could be enhanced by biological interventions.

Conclusion: Although a number of tissue engineering scaffolds have been developed and applied in tendon bone healing, the researches are mainly focused on animal models which are with limitations in clinical application. Since the efficacy and safety of tissue engineering scaffold has been proved, and can be enhanced by biological interventions, substantial clinical trials remain to be done, continued progress in overcoming current tissue engineering challenges should allow for successful clinical practice.

Keywords: bone tissue engineering, scaffold, biomaterials, tendon bone healing, review

\section{IMPACT STATEMENT}

The poor healing of tendon and bone is an important problem that puzzles the postoperative recovery of ligament reconstruction. Usually, after arthroscopic surgery, there is a gap between the graft and bone healing in the bone tunnel. Therefore, researchers hope to obtain better healing effect through other ways such as tissue engineering, so as to improve the function recovery of ligament evaluated the use of issue engineering scaffold especially biomaterials in tendon bone 
and joint postoperatively. In this paper, a systematic summary of the in vivo research was carried out, hoping to summarize the research achievements and find new inspiration and breakthrough to inspire future research.

\section{INTRODUCTION}

Tendon defect is one of the common clinical diseases (Hayashida et al., 2018; Kunze et al., 2019). After the tendon injury, it often leads to limb dysfunction, severe cases, or even disability without repairation in time (Ando et al., 2019). Tendon-bone healing is an important factor in determining the success of ligament reconstruction (Li et al., 2019; Huang et al., 2020). Whether it is knee cruciate ligament, lateral collateral ligament, posterolateral complex reconstruction, shoulder joint sleeve repair, or ankle ligament reconstruction, the degree of tendonbone healing directly affects the postoperative rehabilitation process and surgical effect (Zhao et al., 2019). Studies have shown that in addition to surgical errors, failure to achieve strong tendon-bone healing is the main reason for failure of ligament reconstruction surgery (Han L. et al., 2019; Zhang et al., 2020). Tendon injuries can be divided into two categories: non-defective injuries and defective injuries (Van Der Made et al., 2019; Wisbech Vange et al., 2020). For the tendons with defects and injuries, several treatment methods are available, such as autogenous tendon transplantation, allogeneic tendon transplantation, heterogeneous tendon transplantation, and artificial tendon replacement (Periasamy et al., 2019). In the former, due to lack of donor tendon or immune rejection, the tendon transplantation is restricted. With the development of cell culture technology and transplantation technology and the development of biomaterials science, a new ideal tendon replacement-tissue engineered artificial tendon with synthetic materials, will eventually solve the problem of repairing defective tendons (Khoo and Nikkhah, 2019).

Tissue engineering scaffold mainly serves the following aspects: (1) As a framework connecting cells and tissues, it can be used to guide tissues to grow into a specific shape (LewandowskaLancucka et al., 2015). (2) As a carrier of signaling molecules, it is transported to the defect site, and as a slow-release body, the osteoinductive factor slowly acts. (3) As a place for bone tissue to reproduce, differentiate and metabolize, transport nutrients for cell growth, and eliminate waste. (4) The specific sites on the surface of the scaffold react specifically with the cells and play the role of "identification" and selective adhesion to different types of cells. It can be seen that the scaffold plays an extremely important role in bone tissue engineering, which not only plays a physical role in connecting and supporting cells and tissues but also regulates various functional activities of cells. Tendon/bone tissue engineering has high requirements for scaffold materials, and an ideal in vivo graft should meet the following points. For example, the scaffold materials must be non-toxic and have good biocompatibility (Karel et al., 2018). The material must also be biodegradable and can be gradually degraded and metabolized in the body as the cells proliferate, and then be absorbed (Hejbol et al., 2017). Besides, the degradation products of the materials must be non-toxic, with good biocompatibility, and will not adversely affect tissues and organisms. In addition, the material must have good processing properties and can be processed into the required shape and structure like openpore structure and proper pore size. The scaffold must have good cell affinity, suitability for cell adhesion, proliferation, and secretion of the matrix, as well as certain mechanical properties, including strength and flexibility. Under conventional sterilization conditions, the scaffold must be able to withstand sterilization without physical, chemical, and biological changes. Moreover, the scaffold should not only maintain its shape during the cell culture operation but also be able to withstand the surgical operations implanted in the body, to ensure that it will not break during the operation, can fit with the body, and will not cause mechanical damage to the body tissue.

In recent years, studies on improving tendon-bone healing have mainly focused on promoting ligament-bone integration at tendon sites (Xu et al., 2019). This includes the use of periosteum, biogels, scaffolds, growth factors, stem cells, or other reconstruction materials that promote bone growth or ligament attachment points ( $\mathrm{Uz}$ et al., 2019; Huang et al., 2020; Rodriguez-Vazquez and Ramos-Zuniga, 2020; Sadeghinia et al., 2020). However, reviews describing the application of tissue engineering scaffold, especially biomaterials, in tendonbone healing in vivo are lacking. The purpose of this systematic review was to (1) critique the evidence in vivo regarding the use of tissue engineering scaffold to achieve tendon-bone healing; (2) provide a descriptive summary of the current evidence for tissue engineering scaffold use in tendon-bone healing especially biomaterials, as this is the first systematic review on the topic; and (3) highlight areas of future research to facilitate clinical application.

\section{METHODS}

\section{Eligibility Criteria}

This systematic review was conducted according to the Preferred Reporting Items for Systematic Reviews and Meta-Analyses (PRISMA) guidelines (Maher et al., 2003). This review included original peer-reviewed studies based on the following inclusion criteria: (1) publication in an English-language journal; (2) an in vivo animal study or clinical study that mainly evaluated the use of issue engineering scaffold in tendon bone healing; and (3) scaffold which was manufactured using biomaterials. Studies reporting in vitro work without in vivo analysis were excluded. Only original peer-reviewed articles were included, so letters, editorials, review articles, conference, patents, and meeting abstracts and studies not involving tissue engineering scaffold were excluded.

\section{Literature Search Strategy}

Literature searches were conducted in three electronic databases (PubMed, EMBASE, and Web of Science) from 1 January 1990 to 31 December 2019. The following search terms were used for the literature search: "(tissue engineering OR tissue engineering scaffold OR scaffold for tissue engineering OR scaffold) AND 


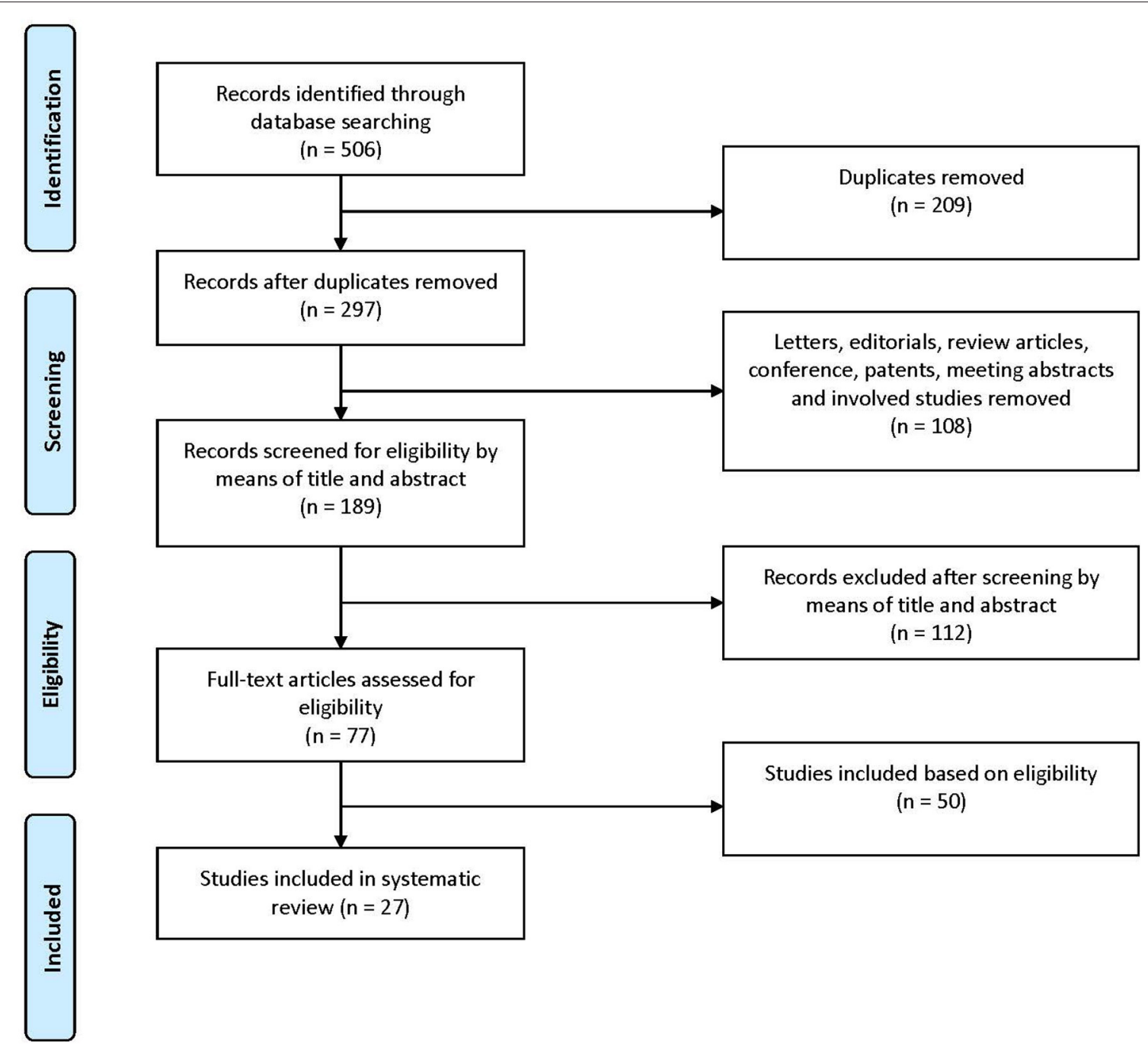

FIGURE 1 | A flowchart showing the selection of studies for inclusion in the systematic review.

(tendon-bone healing OR tendon-bone OR healing of bonetendon) AND (vivo OR human OR patient OR animal OR mouse OR rat OR rabbit OR dog OR sheep OR pig OR horse OR ovine)." Because the scope of this review was large in terms of outcome measures, a systematic review, not a meta-analysis, was performed.

\section{Study Selection}

The articles were initially screened to assess suitability for inclusion according to the criteria with their title and abstract, and then the full text of each article was downloaded to define the relevance of the work for investigators. During selection, the following information was extracted: details of the animal model or clinical information, the groups investigated, the types of scaffold, the methods of evaluation, and the main findings. The selected articles in the study were reviewed, evaluated, and discussed by the authors. A senior investigator would make the final decision if the reviewers were not able to reach a consensus agreement on the inclusion of any articles.

\section{RESULTS}

\section{Search Results}

Figure 1 represents the process for evaluating studies for inclusion in the systematic review. A total of 506 articles were identified through our search. After removal of duplicates, as well as letters, editorials, review articles, conference, patents, meeting abstracts, and involved studies, 189 articles were screened for eligibility by means of title and abstract. Following the exclusion of 112 articles, 77 full-text articles were assessed for eligibility. Finally, 27 studies were identified and included in our analysis, which were consisted of two human trials and 25 animal studies. Furthermore, the 27 studies using this retrieval strategy were analyzed. 


\section{Data Extraction}

Information of data extraction of the 27 studies is available in Table 1, covering details of the animal model or clinical information, the groups investigated, and the types of scaffold. Of the 27 included in vivo studies, two were human trials (Petruskevicius et al., 2002; Mochizuki and Ochi, 2015) and 25 were animal studies. More than half of the animal studies $(n=14)$ utilized a rabbit model (Zhang et al., 2011; Li et al., 2012, 2017; Jiang et al., 2014; Han et al., 2015; Chou et al., 2016; Chung et al., 2017; Lee et al., 2017; Cai et al., 2018; Hu et al., 2018; Chen P. et al., 2019; Han F. et al., 2019; Learn et al., 2019), eight studies used rats (Loeffler et al., 2013; Zhao et al., 2014, 2015a,b; Kovacevic et al., 2015; Zhang et al., 2018; Zhu M. et al., 2019; Zhu Q. et al., 2019), and three studies used pigs (Fleming et al., 2009; Vavken et al., 2012; Li et al., 2014). The involved patients and animals were mature, and no minor or immature animal model had been investigated in previous studies so far as searched. Among the two human trials, only one had tibia defect, which was repaired with Osteoset (Petruskevicius et al., 2002), the other had not point out the defective part (Mochizuki and Ochi, 2015). In animal models, the defect occurred in supraspinatus tendon, infraspinatus tendon, anterior cruciate ligament, long digital extensor tendon, achilles tendon, and proximal tibial metaphysis. Moreover, there were 15 studies that only used synthetic scaffolds like poly lactide-co-glycolide (PLGA), collage, polycaprolactone (PCL), polylactic acid (PLA), and polyethylene terephthalate (PET) to repair the defect of tendon-bone interface, and the rest of the 12 studies used biological interventions, which combined cells or cell factors to enhance the tendon bone healing, such as bone morphogenetic protein (BMP), stromal cell-derived factor (SDF), ligament-derived stem/progenitor cells (LSPCs), kartogenin (KGN), platelet-derived growth factor (PDGF), mesenchymal stem cell (MSC), platelet, etc.

\section{Safety and Efficacy Evaluation}

The adverse events, histological and biomechanical results, and data from other tests were assessed to determine the scaffold safety and efficacy (Table 2). As for the adverse events, no complications were encountered; all the participants and animals recovered well, and macroscopic inspection of the collected specimens did not reveal any gross infectious or inflammatory changes in most of the included studies in vivo. Only one rabbit with self-inflicted wound developed dehiscence and heterotopic of bone formation in the study of Learn et al. (2019), and one animal with minor damage upon tricalcium phosphate (TCP) insertion was found in the study of Li et al. (2014). As for the histological results, it was demonstrated that the tendon-bone integration at the interface using synthetic scaffolds was better than transosseous or direct repair and could be enhanced by biological interventions. The healing was generally characterized by collagen organization improvement, glycosaminoglycan deposition, new bone formation (mineralization), fibroblastlike cells, chondrocyte-like cells, and immature neo-enthesis structure increasement. As for biomechanical tests, the results of ultimate force to failure, stiffness, and strength were extensively investigated and compared, which suggested that the tendonbone healing with scaffolds produced superior biomechanical outcomes to transosseous or direct repair, especially combined with biological interventions. In addition, other tests were performed to evaluate the efficacy of tissue engineering scaffold in tendon bone healing, such as micro-CT, JOA score, and mRNA level, whose results were consistent with the histological results.

\section{DISCUSSION}

With the development of biomaterials science, the tissue engineering scaffold plays an extremely important role in tendonbone healing. This systematic review has proved the efficacy and safety of synthetic scaffolds in vivo, which can be enhanced by biological interventions. However, the previous researches are mainly focused on animal models, not human trials which limits the clinical application of these scaffolds. In this review, we provide a descriptive summary of 27 articles related to the use of tissue engineering scaffold especially biomaterials in tendonbone healing to better guide the clinical practice.

\section{Tendon-Bone Healing Process}

Normal tendon-bone junctions are divided into indirect insertion and direct insertion (Mochizuki et al., 2006, 2014; Iwahashi et al., 2010; Sasaki et al., 2012). Indirect insertion refers to the thick fibrous tissue that directly connects the tendon or ligament to the periosteum, such as the medial collateral ligament tibial point (Mochizuki et al., 2006, 2014; Sasaki et al., 2012). Direct insertion refers to "anchoring" soft tissue to the deep layer of bone through typical fibrous cartilage tissue (Iwahashi et al., 2010). Its direct embedding point is a four-layer tissue transformation area, including bone, calcified cartilage, non-calcified cartilage, and ligament tissues, such as anterior cruciate ligament and rotator cuff. At present, the related research on the surgical technique and fixation method of tendon or ligament reconstruction aims to achieve the maximum contact area between the tendon and bone during reconstruction, ensure the stability and tightness of the contact surface, and minimize the influence of external forces. When the tendon is in contact with the bone, through direct or indirect tendonbone healing, a normal tendon-bone interface is formed, a stable connection is established, and the reconstructed ligament functions. Direct tendon-bone healing refers to a tendon-bone connection with four-layer structure, which exists between the tendon and the tunnel opening after healing (Iwahashi et al., 2010; Sasaki et al., 2012). Indirect tendon-bone healing refers to the connection between tendons and bones through Sharpey-like fibers (Mochizuki et al., 2006, 2014). This type of healing takes a long time, and the tensile strength between tendons and bones after healing is lower than that of direct healing.

An animal experiment showed that (Weiler et al., 2002), after the tendon is implanted in the bone tunnel, it needs to go through the necrosis period, the proliferation period, and the ligamentization period to achieve true healing. The period of necrosis is also called early healing, and generally refers to the first 4 weeks after the implant is implanted. During this process, the graft has necrosis, and its mechanical strength is significantly lower than that of the implant. The proliferative phase refers to the remodeling, revascularization, and strong cell activity of the 
TABLE 1 | The information of data extraction of the included studies.

\begin{tabular}{|c|c|c|c|c|c|c|c|c|}
\hline & References & Species & $\begin{array}{l}\text { Age of } \\
\text { animals } \\
\text { orpatients }\end{array}$ & $\begin{array}{l}\text { Weight of } \\
\text { animals or } \\
\text { patients }\end{array}$ & Type of scafford & $\begin{array}{l}\text { Biological } \\
\text { intervention }\end{array}$ & Group & Defect parts \\
\hline \multirow[t]{2}{*}{ Human studies } & $\begin{array}{l}\text { Mochizuki and } \\
\text { Ochi, } 2015\end{array}$ & Human & $\begin{array}{l}65.5 \text { years } \\
\text { (range: } \\
57-77 \text { years) } \\
\text { old }\end{array}$ & N/A & PGA sheet & None & $\begin{array}{l}\text { PGA group }(n=30) ; P G \text { group }(n \\
=32)\end{array}$ & $\mathrm{N} / \mathrm{A}$ \\
\hline & $\begin{array}{l}\text { Petruskevicius } \\
\text { et al., } 2002\end{array}$ & Human & $19-44$ years & N/A & Osteoset & None & $\begin{array}{l}\text { Osteoset group }(n=10) \text {; Control } \\
\text { group }(n=10)\end{array}$ & Tibia \\
\hline \multirow[t]{7}{*}{ Animal studies } & $\begin{array}{l}\text { Chen C. et al., } \\
2019\end{array}$ & Rabbit & Adult & $2.00-2.20 \mathrm{~kg}$ & PLGA fibrous scaffold & BMP-12 & $\begin{array}{l}\text { Control group }(n=8) \text {; Lenti-BMP } \\
\text { (bone morphogenetic } \\
\text { proteins)-12 group }(n=8)\end{array}$ & $\begin{array}{l}\text { Supraspinatus } \\
\text { tendon }\end{array}$ \\
\hline & $\begin{array}{l}\text { Learn et al., } \\
2019\end{array}$ & $\begin{array}{l}\text { New Zealand } \\
\text { rabbit }\end{array}$ & $\begin{array}{l}8-13 \text { months } \\
\text { old }\end{array}$ & $3-5 \mathrm{~kg}$ & $\begin{array}{l}\text { Electrochemically aligned } \\
\text { collagen (ELAC) }\end{array}$ & MSC & $\begin{array}{l}\text { Direct repair }(n=5) \text {; } \\
\text { Scaffold-alone }(n=5) \text {; } \\
\text { Cell-seeded scaffold repair }(n= \\
\text { 5) }\end{array}$ & $\begin{array}{l}\text { Infraspinatus } \\
\text { tendon in the right } \\
\text { shoulder }\end{array}$ \\
\hline & $\begin{array}{l}\text { Zhu M. et al., } \\
2019\end{array}$ & $\begin{array}{l}\text { Sprague- } \\
\text { Dawley } \\
\text { rat }\end{array}$ & $\begin{array}{l}\geq 12 \text { weeks } \\
\text { old }\end{array}$ & $\geq 350 \mathrm{~g}$ & Synthetic collagen & None & $\begin{array}{l}\text { Sham surgery group }(n=6) ; \\
\text { Unaugmented control group }(n= \\
\text { 20); Intervention group }(n=20)\end{array}$ & $\begin{array}{l}\text { Supraspinatus } \\
\text { tendon }\end{array}$ \\
\hline & $\begin{array}{l}\text { Han F. et al., } \\
2019\end{array}$ & $\begin{array}{l}\text { New Zealand } \\
\text { rabbit }\end{array}$ & 12 weeks old & $2.4 \pm 0.3 \mathrm{~kg}$ & PCL scaffold & $\begin{array}{l}\text { BMP-2; } \\
\text { SDF-1a }\end{array}$ & $\begin{array}{l}\text { PCL group }(n=6) ; \text { B@P group } \\
\text { (PCL scaffold loaded with } \\
\text { BMP-2) }(n=6) ; \mathrm{S}+\text { B@P }(P C L \\
\text { scaffold loaded with BMP-2 and } \\
\text { SDF-1 } 1 \alpha)(n=6)\end{array}$ & $\begin{array}{l}\text { Anterior cruciate } \\
\text { ligament of the } \\
\text { right knee joint }\end{array}$ \\
\hline & $\begin{array}{l}\text { Zhu Q. et al., } \\
2019\end{array}$ & $\begin{array}{l}\text { Sprague- } \\
\text { Dawley } \\
\text { rat }\end{array}$ & 2 months old & $400-500 \mathrm{~g}$ & $\begin{array}{l}\text { PCL fibrous membranes with } \\
\text { aligned nanofibers }\end{array}$ & KGN & $\begin{array}{l}\text { Control group }(n=45) ; P C L \\
\text { group }(n=45) ; K G N-P C L \text { group } \\
\text { (PCL scaffold loaded with } \\
\text { KGN)( }(n=45)\end{array}$ & $\begin{array}{l}\text { Supraspinatus } \\
\text { tendon (superior of } \\
\text { the scapula) }\end{array}$ \\
\hline & $\begin{array}{l}\text { Zhang et al., } \\
2018\end{array}$ & $\begin{array}{l}\text { Mature } \\
\text { female } \\
\text { Sprague- } \\
\text { Dawley } \\
\text { rats }\end{array}$ & N/A & $200-220 \mathrm{~g}$ & $\begin{array}{l}\text { PLLA aligned fiber (A-TSA) } \\
\text { scaffold }\end{array}$ & Trichostatin A & $\begin{array}{l}\text { A-TSA group }(n=7) \text { A groups ( } \\
=7) \text { R-TSA group }(n=7) \mathrm{R} \\
\text { group }(n=7)\end{array}$ & Achilles tendon \\
\hline & Cai et al., 2018 & $\begin{array}{l}\text { New Zealand } \\
\text { rabbit }\end{array}$ & $\begin{array}{l}6-8 \text { months } \\
\text { old }\end{array}$ & $2.8-3.2 \mathrm{~kg}$ & $\begin{array}{l}\text { SF/P(LLA-CL) nanofibrous } \\
\text { scaffolds using silk fibroin } \\
\text { (SF)-blended poly(L-lactic } \\
\text { acid-co-e-caprolactone) } \\
\text { (PLLA-CL) }\end{array}$ & None & $\begin{array}{l}\text { Random scaffold (RS) }(n=30) \text {; } \\
\text { Dual-layer aligned-random } \\
\text { nanofibrous scaffolds(ARS) }(n= \\
\text { 30); Control groups }(n=30)\end{array}$ & $\begin{array}{l}\text { Achilles tendon of } \\
\text { one hindlimb }\end{array}$ \\
\hline
\end{tabular}


TABLE 1 | Continued

\begin{tabular}{|c|c|c|c|c|c|c|c|}
\hline References & Species & $\begin{array}{l}\text { Age of } \\
\text { animals } \\
\text { orpatients }\end{array}$ & $\begin{array}{l}\text { Weight of } \\
\text { animals or } \\
\text { patients }\end{array}$ & Type of scafford & $\begin{array}{l}\text { Biological } \\
\text { intervention }\end{array}$ & Group & Defect parts \\
\hline Hu et al., 2018 & $\begin{array}{l}\text { New Zealand } \\
\text { rabbit }\end{array}$ & $\mathrm{N} / \mathrm{A}$ & $2.5-3.0 \mathrm{~kg}$ & $\begin{array}{l}\text { Collage-silk; SDF-1-releasing } \\
\text { collagen-silk (CSF) scaffold }\end{array}$ & $\begin{array}{l}\text { SDF-1; } \\
\text { LSPCs }\end{array}$ & $\begin{array}{l}\text { CS (collage-silk) ( } n=10) \text {; CSL } \\
\text { (collage-silk with LSPCs } \\
\text { injection) ( } n=15) ; \text { CSF } \\
\text { (collage-silk-SDF-1) }(n=10) ; \\
\text { CSFL (collage-silk-SDF-1 with } \\
\text { LSPCs injection) }(n=15) ;\end{array}$ & $\begin{array}{l}\text { Anterior cruciate } \\
\text { ligament of the } \\
\text { right knee joint }\end{array}$ \\
\hline Li et al., 2017 & $\begin{array}{l}\text { New Zealand } \\
\text { rabbit }\end{array}$ & $\begin{array}{l}6-8 \text { months } \\
\text { old }\end{array}$ & $2.8-3.5 \mathrm{~kg}$ & $\begin{array}{l}\text { Simplex fibrous membrane of } \\
\text { PLLA; Dual-layer } \\
\text { organic/inorganic flexible bipolar } \\
\text { fibrous membrane (nHA-PLLA) }\end{array}$ & None & $\begin{array}{l}\text { Control group ( } n=48) \text {; Simplex } \\
\text { fibrous membrane of PLLA (SFM) } \\
\text { group }(n=48) \text {; dual-layer } \\
\text { organic/inorganic flexible bipolar } \\
\text { fibrous membrane (BFM) group } \\
(n=48)\end{array}$ & $\begin{array}{l}\text { Supraspinatus } \\
\text { tendon }\end{array}$ \\
\hline $\begin{array}{l}\text { Chung et al., } \\
2017\end{array}$ & $\begin{array}{l}\text { New Zealand } \\
\text { rabbit }\end{array}$ & $\begin{array}{l}6-7 \text { months } \\
\text { old }\end{array}$ & $3.2-3.5 \mathrm{~kg}$ & $\begin{array}{l}\text { A biodegradable and synthetic } \\
\text { tri-component graft consisting of } \\
\text { porous } \\
\text { poly(1,8-octanediol-co-citric } \\
\text { acid)-hydroxyapatite } \\
\text { nanocomposites (POC-HA) and } \\
\text { poly(l-lactide) (PLL) braids }\end{array}$ & None & $\begin{array}{l}\text { Reconstructed animals }(n=3) \text {; } \\
\text { Non-operated control animals ( } n \\
=3 \text { ) }\end{array}$ & $\begin{array}{l}\text { Anterior cruciate } \\
\text { ligament of the } \\
\text { right knee joint }\end{array}$ \\
\hline Lee et al., 2017 & $\begin{array}{l}\text { New Zealand } \\
\text { rabbit }\end{array}$ & Adult & $3.0-3.5 \mathrm{~kg}$ & $\begin{array}{l}\text { Recombinant human } \\
\text { rhBMP-2-containing collagen gel }\end{array}$ & rhBMP-2 & $\begin{array}{l}\text { Saline only (control) }(n=12) ; \\
\text { Collagen gel without rhBMP-2 }(n \\
=12) ; \text { rhBMP-2-conjugated } \\
\text { collagen gel }(n=12)\end{array}$ & $\begin{array}{l}\text { Long digital } \\
\text { extensor tendon }\end{array}$ \\
\hline $\begin{array}{l}\text { Chou et al., } \\
2016\end{array}$ & $\begin{array}{l}\text { New Zealand } \\
\text { rabbit }\end{array}$ & Adult & $3.3 \pm 0.7 \mathrm{~kg}$ & $\begin{array}{l}\text { PLA; PLGA/collagen nanofibrous } \\
\text { membrane }\end{array}$ & None & $\begin{array}{l}\text { Control }(n=24) \text {; PLGA/collagen } \\
\text { nanofibrous membrane }(n=24)\end{array}$ & $\begin{array}{l}\text { Long digital } \\
\text { extensor tendon }\end{array}$ \\
\hline Han et al., 2015 & $\begin{array}{l}\text { New Zealand } \\
\text { rabbits }\end{array}$ & 12 weeks old & $2.4 \pm 0.3 \mathrm{~kg}$ & $\begin{array}{l}\text { A biomimetic nanofiber } \\
\text { membrane of polycaprolactone/ } \\
\text { nanohydroxyapatite/collagen } \\
\text { (PCL/nHAp/Col) }\end{array}$ & None & $\begin{array}{l}\mathrm{PCL} / \mathrm{nHAp} / \mathrm{Col} \text { group }(n=6) ; \\
\mathrm{PCL} \text { group }(n=6)\end{array}$ & $\begin{array}{l}\text { Anterior cruciate } \\
\text { ligament of the } \\
\text { right knee joint }\end{array}$ \\
\hline Bi et al., 2015 & $\begin{array}{l}\text { New Zealand } \\
\text { rabbit }\end{array}$ & 13 weeks old & $2.5-3.0 \mathrm{~kg}$ & Silk-collagen scaffold & None & $\begin{array}{l}\text { Scaffold group }(n=20) ; \\
\text { Autograft group }(n=20)\end{array}$ & $\begin{array}{l}\text { Anterior cruciate } \\
\text { ligament of the } \\
\text { right knee joint }\end{array}$ \\
\hline $\begin{array}{l}\text { Kovacevic et al., } \\
2015\end{array}$ & $\begin{array}{l}\text { Sprague- } \\
\text { Dawley } \\
\text { rat }\end{array}$ & $\mathrm{N} / \mathrm{A}$ & N/A & Collagen scaffold & PDGF & $\begin{array}{l}\text { Control }(n=19) ; \text { Scaffold only }(n \\
=19) ; \text { Three PDGF doses ( } 30 \\
\mu \mathrm{g} / \mathrm{mol} / \mathrm{L}, 100 \mu \mathrm{g} / \mathrm{mol} / \mathrm{L}, 300 \\
\mu \mathrm{g} / \mathrm{mol} / \mathrm{L} \text { ) on the collagen } \\
\text { scaffold }(n=19)\end{array}$ & $\begin{array}{l}\text { Supraspinatus } \\
\text { tendon }\end{array}$ \\
\hline $\begin{array}{l}\text { Zhao et al., } \\
2015 a\end{array}$ & $\begin{array}{l}\text { Sprague- } \\
\text { Dawley } \\
\text { rat }\end{array}$ & $\mathrm{N} / \mathrm{A}$ & $350-400 \mathrm{~g}$ & $\begin{array}{l}\text { PCL scaffold; Scaffold } \\
\text { composed of microfibers of PCL } \\
\text { and nanofibers of CS }\end{array}$ & None & $\begin{array}{l}\text { Transosseous repair }(n=48) \text {; } \\
\text { PCL scaffolds }(n=48) \text { PCL-CS } \\
\text { scaffolds ( } n=48)\end{array}$ & $\begin{array}{l}\text { Left supraspinatus } \\
\text { tendon }\end{array}$ \\
\hline
\end{tabular}


TABLE 1| Continued

\begin{tabular}{|c|c|c|c|c|c|c|c|}
\hline References & Species & $\begin{array}{l}\text { Age of } \\
\text { animals } \\
\text { orpatients }\end{array}$ & $\begin{array}{l}\text { Weight of } \\
\text { animals or } \\
\text { patients }\end{array}$ & Type of scafford & $\begin{array}{l}\text { Biological } \\
\text { intervention }\end{array}$ & Group & Defect parts \\
\hline $\begin{array}{l}\text { Zhao et al., } \\
2015 b\end{array}$ & $\begin{array}{l}\text { Sprague- } \\
\text { Dawley } \\
\text { rat }\end{array}$ & $\mathrm{N} / \mathrm{A}$ & $350-401 \mathrm{~g}$ & $\begin{array}{l}\text { PLLA fibrous membranes; } \\
\text { Gelatin-PLLA }\end{array}$ & None & $\begin{array}{l}\text { Transosseous repair }(n=48) \text {; } \\
\text { PLLA membranes }(n=48) \text {; } \\
\text { Gelatin-PLLA membranes ( } n= \\
\text { 48) }\end{array}$ & $\begin{array}{l}\text { Left supraspinatus } \\
\text { tendon }\end{array}$ \\
\hline Li et al., 2014 & Pig & 3 months old & 47 and $52 \mathrm{~kg}$ & $\begin{array}{l}\text { Silk-TCP-PEEK scaffold of silk, } \\
\text { TCP, and PEEK }\end{array}$ & None & N/A & $\begin{array}{l}\text { Anterior cruciate } \\
\text { ligament of the } \\
\text { knee joint }\end{array}$ \\
\hline $\begin{array}{l}\text { Jiang et al., } \\
2014\end{array}$ & $\begin{array}{l}\text { New Zealand } \\
\text { rabbit }\end{array}$ & Adult & $2.8 \pm 0.5 \mathrm{~kg}$ & PET & None & $\begin{array}{l}\text { PET group }(n=24) ; \text { PET }+ \text { SF } \\
\text { group (SF-coating group) }(n= \\
\text { 24); PET }+ \text { SF }+ \text { HAP group } \\
\text { (combined HAP- and SF-coating } \\
\text { group) }(n=24)\end{array}$ & $\begin{array}{l}\text { Proximal tibial } \\
\text { metaphysis }\end{array}$ \\
\hline Zhao et al., 2014 & $\begin{array}{l}\text { Sprague- } \\
\text { Dawley } \\
\text { rat }\end{array}$ & N/A & $350-400 \mathrm{~g}$ & PLGA membranes & bFGF & $\begin{array}{l}\text { Transosseous repair }(n=48) \text {; } \\
\text { PLGA membranes }(n=48) \text {; } \\
\text { bFGF-PLGA membranes }(n= \\
\text { 48) }\end{array}$ & $\begin{array}{l}\text { Left supraspinatus } \\
\text { tendon }\end{array}$ \\
\hline $\begin{array}{l}\text { Loeffler et al., } \\
2013\end{array}$ & Lewis rat & $N / A$ & $\begin{array}{l}13.8 \text { weeks } \pm \\
2 / 7\end{array}$ & Sponge & $\begin{array}{l}\text { Cultured cells } \\
\text { from the } \\
\text { tendon-to- } \\
\text { bone } \\
\text { interface }\end{array}$ & $\begin{array}{l}\text { Group I (control without surgery); } \\
\text { Group II (surgical defect in the } \\
\text { rotator cuff only); Group III } \\
\text { (surgical defect with suture repair } \\
\text { only); Group IV (surgical defect } \\
\text { and repair with sponge only); } \\
\text { Group V (surgical defect and } \\
\text { repair with sponge loaded with } \\
\text { cells) }\end{array}$ & $\begin{array}{l}\text { Supraspinatus } \\
\text { tendon }\end{array}$ \\
\hline $\begin{array}{l}\text { Vavken et al., } \\
2012\end{array}$ & Yorkshire pig & $\begin{array}{l}11.8 \pm 0.4 \\
\text { weeks old }\end{array}$ & $30 \pm 1.1 \mathrm{~kg}$ & Collagen Scaffold & Platelet & $\begin{array}{l}\text { ACL reconstruction }(n=8) ; \\
\text { Enhanced } \mathrm{ACL} \text { repair }(n=8) ; \\
\text { ACL transection }(n=8) ;\end{array}$ & $\begin{array}{l}\text { Anterior cruciate } \\
\text { ligament of the } \\
\text { knee joint }\end{array}$ \\
\hline Li et al., 2012 & $\begin{array}{l}\text { New Zealand } \\
\text { rabbit }\end{array}$ & Adult & $\mathrm{N} / \mathrm{A}$ & PET artificial ligament & None & Control group; LBL group & $\begin{array}{l}\text { Anterior cruciate } \\
\text { ligament of the } \\
\text { knee joint }\end{array}$ \\
\hline $\begin{array}{l}\text { Zhang et al., } \\
2011\end{array}$ & $\begin{array}{l}\text { New Zealand } \\
\text { rabbit }\end{array}$ & 22 weeks old & $2.7 \pm 0.2 \mathrm{~kg}$ & Fibrin glue-BMP & BMP & $\begin{array}{l}\text { Control group }(n=17) \text {; Fibrin } \\
\text { glue-BMP group }(n=17) \text {; RBX } \\
\text { (recombined bone xenograft) } \\
\text { group }(n=17)\end{array}$ & $\begin{array}{l}\text { Long digital } \\
\text { extensor tendon }\end{array}$ \\
\hline $\begin{array}{l}\text { Fleming et al., } \\
2009\end{array}$ & Yorkshire pig & $N / A$ & $30 \mathrm{~kg}$ & $\begin{array}{l}\text { Collagen-platelet composite } \\
\text { (CPC) }\end{array}$ & None & $\begin{array}{l}\text { Control group }(n=7) \text {; } \\
\text { Experimental Group }(n=7)\end{array}$ & $\begin{array}{l}\text { Anterior cruciate } \\
\text { ligament of the } \\
\text { knee joint }\end{array}$ \\
\hline
\end{tabular}

PGA, polyglycolic acid; BMP, bone morphogenetic protein; PCL, polycaprolactone; SDF, stromal cell-derived factor; PDGF, platelet-derived growth factor; PLLA, poly I-lactide; TCP, tricalcium phosphate; ELAC, electrochemically aligned collagen; PLA, polylactic acid; SF, silk fibroin; PLGA, poly lactide-co-glycolide; MSC, mesenchymal stem cell; KGN, kartogenin; LSPCs, ligament-derived stem/progenitor cells; CS, chitosan; PET, polyethylene terephthalate; PEEK, polyether ether ketone; ACL, anterior cruciate ligament; HAp/HA, hydroxyapatite. 
TABLE 2 | The safety and efficacy of tissue engineering scaffold in tendon bone healing in vivo.

\begin{tabular}{|c|c|c|c|c|c|c|c|}
\hline & References & Group & Follow-up & $\begin{array}{l}\text { Adverse } \\
\text { events }\end{array}$ & Histological data & Biomechanical data & Data from other tests \\
\hline \multirow[t]{2}{*}{$\begin{array}{l}\text { Human } \\
\text { studies }\end{array}$} & $\begin{array}{l}\text { Mochizuki and } \\
\text { Ochi, } 2015\end{array}$ & $\begin{array}{l}\text { PGA group }(n=30) ; P G \\
\text { group }(n=32) ;\end{array}$ & $\begin{array}{l}1,3,6,12 \\
\text { months }\end{array}$ & None & N/A & N/A & $\begin{array}{l}\text { The mean JOA scores of PGA } \\
\text { group and PG group improved } \\
\text { and the high-intensity rate was } \\
\text { significantly lower for the PGA } \\
\text { group by MRI }\end{array}$ \\
\hline & $\begin{array}{l}\text { Petruskevicius } \\
\text { et al., } 2002\end{array}$ & $\begin{array}{l}\text { Osteoset group }(n=10) \\
\text { Control group }(n=10)\end{array}$ & $\begin{array}{l}6 \text { weeks, } \\
3,6 \\
\text { months }\end{array}$ & None & N/A & N/A & $\begin{array}{l}\text { The same amount of bone in the } \\
\text { defect was found in the Osteoset } \\
\text { and control groups, but the bone } \\
\text { volume increased in the control } \\
\text { group, and the Osteoset pellets } \\
\text { were almost resorbed after } 6 \\
\text { weeks }\end{array}$ \\
\hline \multirow[t]{4}{*}{$\begin{array}{l}\text { Animal } \\
\text { studies }\end{array}$} & $\begin{array}{l}\text { Chen C. et al., } \\
2019\end{array}$ & $\begin{array}{l}\text { Control group }(n=8) \\
\text { Lenti-BMP (bone } \\
\text { morphogenetic proteins)-12 } \\
\text { group }(n=8)\end{array}$ & $\begin{array}{l}4,8,12 \\
\text { weeks }\end{array}$ & N/A & $\begin{array}{l}\text { Application of BMP-12 overexpressing } \\
\text { BM-MSCs-loaded PLGA scaffolds } \\
\text { increased the amount of fibrocartilage } \\
\text { formation and improve the collagen fiber } \\
\text { organization at the interface between the } \\
\text { tendon and bone }\end{array}$ & $\begin{array}{l}\text { The ultimate force to failure in the } \\
\text { Ad-BMP-12 group was } \\
\text { significantly higher than that in } \\
\text { control group }\end{array}$ & $\begin{array}{l}\text { The Ad-BMP-12 group had } \\
\text { significantly higher modified } \\
\text { tendon maturing scores than the } \\
\text { control group at } 4,8 \text {, and } 12 \\
\text { weeks post-surgery }\end{array}$ \\
\hline & $\begin{array}{l}\text { Learn et al., } \\
2019\end{array}$ & $\begin{array}{l}\text { Direct repair }(n=5) \\
\text { Scaffold-alone }(n=5) \text {; } \\
\text { Cell-seeded scaffold repair } \\
(n=5)\end{array}$ & 3 months & $\begin{array}{l}\text { One rabbit } \\
\text { with self- } \\
\text { inflicted } \\
\text { wound } \\
\text { dehiscence } \\
\text { and } \\
\text { heterotopic } \\
\text { bone } \\
\text { formation }\end{array}$ & $\begin{array}{l}\text { Robust collagen deposition around ELAC } \\
\text { fibers and increased cellularity within the } \\
\text { continuum of woven scaffolds as } \\
\text { compared to native tendon }\end{array}$ & $\begin{array}{l}\text { The maximum load-bearing } \\
\text { capacity was comparable } \\
\text { between all groups, while } \\
\text { MSC-seeded scaffold repairs } \\
\text { exhibited increased stiffness } \\
\text { relative to non-seeded scaffold } \\
\text { repairs }\end{array}$ & $\begin{array}{l}\text { Immunohistochemical staining } \\
\text { revealed presence of collagens I } \\
\text { and III in all groups, but } \\
\text { procollagen I and the } \\
\text { tendon-specific marker } \\
\text { tenomodulin were only observed } \\
\text { in seeded and non-seeded } \\
\text { ELAC scaffold repairs }\end{array}$ \\
\hline & $\begin{array}{l}\text { Zhu M. et al., } \\
2019\end{array}$ & $\begin{array}{l}\text { Sham surgery group }(n=6) ; \\
\text { Unaugmented control group } \\
(n=20) ; \text { Intervention group } \\
(n=20)\end{array}$ & $\begin{array}{l}6,12 \\
\text { weeks }\end{array}$ & None & $\begin{array}{l}\text { Improved collagen fiber density and } \\
\text { orientation scores in the tendon and } \\
\text { improved enthesis with early formation of a } \\
\text { fibrocartilage transition zone were } \\
\text { observed in the scaffold group }\end{array}$ & $\begin{array}{l}\text { No significant difference was } \\
\text { detected in Biomechanical } \\
\text { analysis }\end{array}$ & $N / A$ \\
\hline & $\begin{array}{l}\text { Han F. et al., } \\
2019\end{array}$ & $\begin{array}{l}\text { PCL group }(n=6) ; \text { B@P } \\
\text { group (PCL scaffold loaded } \\
\text { with BMP-2) }(n=6) ; \mathrm{S}+ \\
\text { B@P (PCL scaffold loaded } \\
\text { with BMP-2 and SDF-1 } \alpha)(n \\
=6)\end{array}$ & 4,8 weeks & N/A & $\begin{array}{l}\text { Thin fibrous scar tissue in the tendon-bone } \\
\text { interface and a large amount of new bone } \\
\text { inside the autograft were formed in the S } \\
\text { + B@P group. In the B@P group, the } \\
\text { fibrous scar tissue was thin, and there was } \\
\text { less new bone formation at the interface. } \\
\text { In the PCL group, there was still more } \\
\text { fibrous scar tissue at the interface, no } \\
\text { significant mineralization was observed, } \\
\text { and many membranes remained. }\end{array}$ & $\begin{array}{l}\text { The S + B@P group exhibited } \\
\text { superior mechanical properties } \\
\text { compared to those the B@P and } \\
\text { PCL groups, and had a higher } \\
\text { failure force and stiffness }\end{array}$ & $\begin{array}{l}\text { Immunohistochemical staining } \\
\text { revealed that the secretion of } \\
\text { OCN and OPN in the bone } \\
\text { tunnel always remained at a high } \\
\text { level in the S + B@P group }\end{array}$ \\
\hline
\end{tabular}

One rabbit Robust collagen depostion around ELAC coes and increased cellularity within the

dehiscence

and

bone

Application of BMP-12 overexpressing

MSCs-loaded PLGA scaffolds

The ultimate force to failure in the

Ad-BMP-12 group was

conificantly higher than that in

The maximum load-bearing

was comparable

MSC-seeded scaffold repairs

exhibited increased stiffness

relative to non-seeded scaffold

PGA

group and $P G$ group improved significantly lower for the PGA

group by MRI

defect was found in the Osteoset

groups, but the bone

increased in the control

, and the Osteoset pellets

tendon maturing scores than the control group at 4, 8, and 12

mmunohistochemical staining of collagens ।

tenomodulin were only observed in seeded and non-seeded

scaffold repairs 
TABLE 2 | Continued

\begin{tabular}{|c|c|c|c|c|c|c|}
\hline References & Group & Follow-up & $\begin{array}{l}\text { Adverse } \\
\text { events }\end{array}$ & Histological data & Biomechanical data & Data from other tests \\
\hline $\begin{array}{l}\text { Zhu Q. et al., } \\
2019\end{array}$ & $\begin{array}{l}\text { Control group }(n=45) ; P C L \\
\text { group }(n=45) ; K G N-P C L \\
\text { group (PCL scaffold loaded } \\
\text { with KGN) }(n=45)\end{array}$ & $\begin{array}{l}2,4,8 \\
\text { weeks }\end{array}$ & N/A & $\begin{array}{l}\text { KGN-PCL membranes promoted } \\
\text { fibrocartilage formation and collagen } \\
\text { organization }\end{array}$ & $\begin{array}{l}\text { The ultimate load to failure in the } \\
\text { PCL-KGN group was highest } \\
\text { among the three groups, and } \\
\text { that in PCL group was higher } \\
\text { than that in the control group }\end{array}$ & N/A \\
\hline $\begin{array}{l}\text { Zhang et al., } \\
2018\end{array}$ & $\begin{array}{l}\text { A-TSA group }(n=7) \text { A } \\
\text { groups }(n=7) \text { R-TSA group } \\
(n=7) \text { R group }(n=7)\end{array}$ & 2,4 weeks & N/A & $\begin{array}{l}\text { The formation of regenerated tendon with } \\
\text { the typical structure of tendon at the } \\
\text { repaired site in the A-TSA and A treated } \\
\text { groups. R-TSA treated group had a much } \\
\text { better histological structure compared with } \\
\text { R treated group. }\end{array}$ & $\begin{array}{l}\text { The A-TSA group had better } \\
\text { biomechanical properties than } \\
\text { other three groups }\end{array}$ & $\begin{array}{l}\text { The mRNA expression of Mkx } \\
\text { was upregulated } \sim 2.3 \text {-fold in } \\
\text { A-TSA treated group. The } \\
\text { average diameter of collagen } \\
\text { fibrils in the A-TSA group ( } 50.48 \\
\pm 10 \mathrm{~nm} \text { ) was larger than other } \\
\text { groups. }\end{array}$ \\
\hline Cai et al., 2018 & $\begin{array}{l}\text { Random scaffold (RS) }(n= \\
\text { 30); Dual-layer } \\
\text { aligned-random nanofibrous } \\
\text { scaffolds(ARS) }(n=30) ; \\
\text { Control groups }(n=30)\end{array}$ & $\begin{array}{l}6,12 \\
\text { weeks }\end{array}$ & N/A & $\begin{array}{l}\text { The ARS significantly increased the area of } \\
\text { metachromasia, decreased the interface } \\
\text { width, and improved collagen maturation } \\
\text { and organization at the tendon-bone } \\
\text { interface compared with the RS and } \\
\text { control groups }\end{array}$ & $\begin{array}{l}\text { The ARS groups had a better } \\
\text { ultimate load-to-failure and } \\
\text { stiffness than the RS and control } \\
\text { groups }\end{array}$ & $\begin{array}{l}\text { Micro-CT showed that the bone } \\
\text { tunnel area of RS and ARS } \\
\text { groups was significantly smaller } \\
\text { than those of the control group; } \\
\text { Real-time polymerase chain } \\
\text { reaction showed that BMP-2 and } \\
\text { osteopontin expression levels at } \\
\text { the interface in the RS and ARS } \\
\text { groups were higher than those of } \\
\text { the control group, and collagen I } \\
\text { expression level of the ARS } \\
\text { group was significantly higher } \\
\text { than those of the RS and control } \\
\text { groups. }\end{array}$ \\
\hline Hu et al., 2018 & $\begin{array}{l}\text { CS (collage-silk) }(n=10) ; \\
\text { CSL (collage-silk with } \\
\text { LSPCs injection) }(n=15) ; \\
\text { CSF (collage-silk-SDF-1) }(n \\
=10) ; \text { CSFL } \\
\text { (collage-silk-SDF-1 with } \\
\text { LSPCs injection) }(n=15) ;\end{array}$ & & N/A & $\begin{array}{l}\text { The CSFL group exhibited enhanced } \\
\text { maturation of ACL tissue and improved } \\
\text { bone tunnel healing }\end{array}$ & N/A & N/A \\
\hline Li et al., 2017 & $\begin{array}{l}\text { Control group ( } n=48 \text { ); } \\
\text { Simplex fibrous membrane } \\
\text { of PLLA (SFM) group ( } n= \\
\text { 48); dual-layer } \\
\text { organic/inorganic flexible } \\
\text { bipolar fibrous membrane } \\
\text { (BFM) group ( } n=48 \text { ) }\end{array}$ & $\begin{array}{l}4,8,12 \\
\text { weeks }\end{array}$ & None & $\begin{array}{l}\text { BFM significantly increased the area of } \\
\text { glycosaminoglycan staining at the } \\
\text { tendon-bone interface and improved } \\
\text { collagen organization when compared to } \\
\text { the simplex fibrous membrane (SFM) of } \\
\text { PLLA. }\end{array}$ & $\begin{array}{l}\text { The BFM group had a greater } \\
\text { ultimate load-to-failure and } \\
\text { stiffness than the SFM group at } \\
12 \text { weeks after surgery }\end{array}$ & $\begin{array}{l}\text { Implanting the bipolar membrane } \\
\text { also induced bone formation and } \\
\text { fibrillogenesis as assessed by } \\
\text { micro-CT. }\end{array}$ \\
\hline $\begin{array}{l}\text { Chung et al., } \\
2017\end{array}$ & $\begin{array}{l}\text { Reconstructed animals }(n= \\
\text { 3); Non-operated control } \\
\text { animals }(n=3)\end{array}$ & 6 weeks & N/A & $\begin{array}{l}\text { In reconstructed animals, tissue infiltration } \\
\text { throughout the entire scaffold and tissue } \\
\text { ingrowth and interlocking within the bone } \\
\text { tunnels. }\end{array}$ & N/A & N/A \\
\hline
\end{tabular}




\begin{tabular}{|c|c|c|c|c|c|c|}
\hline References & Group & Follow-up & $\begin{array}{l}\text { Adverse } \\
\text { events }\end{array}$ & Histological data & Biomechanical data & Data from other tests \\
\hline Lee et al., 2017 & $\begin{array}{l}\text { Saline only (control) }(n= \\
\text { 12); Collagen gel without } \\
\text { rhBMP-2( } n=12) ; \\
\text { rhBMP-2-conjugated } \\
\text { collagen gel( }(n=12)\end{array}$ & 3,6 weeks & N/A & $\begin{array}{l}\text { Fibrocartilage and new bone are formed at } \\
\text { the interface at } 6 \text { weeks after injection of } \\
\text { rhBMP-2 }\end{array}$ & N/A & $\begin{array}{l}\text { The micro-CT scan showed that } \\
\text { spotty calcification appeared and } \\
\text { enthesis-like tissue was } \\
\text { produced successfully in the } \\
\text { tendon at } 6 \text { weeks after injection } \\
\text { of rhBMP-2 }\end{array}$ \\
\hline $\begin{array}{l}\text { Chou et al., } \\
2016\end{array}$ & $\begin{array}{l}\text { Control }(n=24) \text {; } \\
\text { PLGA/collagen nanofibrous } \\
\text { membrane }(n=24)\end{array}$ & 16 weeks & N/A & $\begin{array}{l}\text { The adequate biocompatibility of the PLA } \\
\text { bolt on a medial cortex with progressive } \\
\text { bone ingrowth and without tissue } \\
\text { overreaction }\end{array}$ & $\begin{array}{l}\text { The average maximal failure } \\
\text { loads in PLGA/collagen } \\
\text { nanofibrous membrane group } \\
\text { was significantly higher than that } \\
\text { in cintrol group }\end{array}$ & $\mathrm{N} / \mathrm{A}$ \\
\hline Han et al., 2015 & $\begin{array}{l}\mathrm{PCL} / \mathrm{nHAp} / \mathrm{Col} \text { group }(n= \\
\text { 6); } \mathrm{PCL} \text { group }(n=6)\end{array}$ & 4,8 weeks & N/A & $\begin{array}{l}\text { The scar tissue thickness was clearly } \\
\text { smaller in the PCL/nHAp/Col group } \\
\text { compared with the control group, and new } \\
\text { bone tissue could be seen at the interface } \\
\text { in PCL/nHAp/Col group }\end{array}$ & $\begin{array}{l}\text { The failure load and the average } \\
\text { stiffness was significantly higher } \\
\text { in PCL/nHAp/Col group than in } \\
\text { PCL control group }\end{array}$ & N/A \\
\hline Bi et al., 2015 & $\begin{array}{l}\text { Scaffold group }(n=20) \\
\text { Autograft group }(n=20)\end{array}$ & $\begin{array}{l}4,16 \\
\text { weeks }\end{array}$ & None & $\begin{array}{l}\text { Abundant fibroblast-like cells were found } \\
\text { in the core of the scaffold graft, and } \\
\text { tenascin-C was strongly positive in newly } \\
\text { regenerated tissu in the scaffold group, } \\
\text { similar to the autograft group. }\end{array}$ & $\begin{array}{l}\text { The failure load in the scaffold } \\
\text { group was significantly higher } \\
\text { than that in the autograft group } \\
\text { at } 4 \text { weeks postoperatively. At } \\
\text { week } 16 \text {, the stiffness in scaffold } \\
\text { group was significantly greater } \\
\text { than that of the autograft group }\end{array}$ & $\begin{array}{l}\text { Micro-CT scan found that } \\
\text { obvious signals suggesting newly } \\
\text { formed mineralized tissue were } \\
\text { detected in the bone tunnels of } \\
\text { both groups, and the average } \\
\text { bone tunnel area in the scaffold } \\
\text { group was significantly smaller } \\
\text { than that in the autograft group }\end{array}$ \\
\hline $\begin{array}{l}\text { Kovacevic et al., } \\
2015\end{array}$ & $\begin{array}{l}\text { Control }(n=19) \text {; Scaffold } \\
\text { only }(n=19) ; \text { Three PDGF } \\
\text { doses (30, } 100,300 \\
\mu \mathrm{g} / \mathrm{mol} / \mathrm{L}) \text { on the collagen } \\
\text { scaffold }(n=19)\end{array}$ & 5,28 days & None & $\begin{array}{l}\text { rhPDGF-BB delivery on a scaffold } \\
\text { demonstrated a dose-dependent } \\
\text { response in cellular proliferation and } \\
\text { angiogenesis compared with the control } \\
\text { and scaffold groups at } 5 \text { days, and had no } \\
\text { effect on increasing fibrocartilage } \\
\text { formation or improving collagen fiber } \\
\text { maturity compared with controls at } 28 \\
\text { days. }\end{array}$ & $\begin{array}{l}\text { The control group had higher } \\
\text { tensile loads to failure and } \\
\text { stiffness than all the groups } \\
\text { receiving the scaffold }\end{array}$ & $\mathrm{N} / \mathrm{A}$ \\
\hline $\begin{array}{l}\text { Zhao et al., } \\
2015 a\end{array}$ & $\begin{array}{l}\text { Transosseous repair ( } n= \\
\text { 48); PCL scaffolds }(n=48) \\
\text { PCL-CS scaffolds ( } n=48 \text { ) }\end{array}$ & $\begin{array}{l}2,4,8 \\
\text { weeks }\end{array}$ & N/A & $\begin{array}{l}\text { The PCL-CS scaffolds enhanced new } \\
\text { bone formation (mineralization) and } \\
\text { collagen and glycosaminoglycan } \\
\text { expression (major components of } \\
\text { extracellular matrix) compared to the PCL } \\
\text { scaffolds }\end{array}$ & $\begin{array}{l}\text { The torn tissues at the } \\
\text { tendon-bone insertion site } \\
\text { regenerated with the PCL-CS } \\
\text { scaffolds showed higher } \\
\text { strength, failure strain and } \\
\text { stiffness compared to those } \\
\text { repaired using only the PCL } \\
\text { scaffolds }\end{array}$ & $\mathrm{N} / \mathrm{A}$ \\
\hline $\begin{array}{l}\text { Zhao et al., } \\
2015 b\end{array}$ & $\begin{array}{l}\text { Transosseous repair }(n= \\
\text { 48); PLLA membranes ( } n= \\
\text { 48); Gelatin-PLLA } \\
\text { membranes ( } n=48)\end{array}$ & $\begin{array}{l}2,4,8 \\
\text { weeks }\end{array}$ & None & $\begin{array}{l}\text { Gelatin-PLLA membranes have excellent } \\
\text { biocompatibility and biodegradability, and } \\
\text { significantly increased the area of } \\
\text { glycosaminoglycan staining and improved } \\
\text { collagen organization compared with the } \\
\text { control group }\end{array}$ & $\begin{array}{l}\text { Gelatin-PLLA group had a } \\
\text { greater ultimate load to failure } \\
\text { and stiffness than the control } \\
\text { group }\end{array}$ & $\mathrm{N} / \mathrm{A}$ \\
\hline
\end{tabular}




\begin{tabular}{|c|c|c|c|c|c|c|}
\hline References & Group & Follow-up & $\begin{array}{l}\text { Adverse } \\
\text { events }\end{array}$ & Histological data & Biomechanical data & Data from other tests \\
\hline Li et al., 2014 & $\mathrm{~N} / \mathrm{A}$ & 3 months & $\begin{array}{l}\text { One animal } \\
\text { with minor } \\
\text { damage } \\
\text { upon TCP } \\
\text { insertion }\end{array}$ & $\begin{array}{l}\text { There was a robust histological transition } \\
\text { between regenerated fibrous tissue and } \\
\text { the margins of the bone tunnel in } \\
\text { Silk-TCP-PEEK, which had histological } \\
\text { features similar to the native ACL to bone } \\
\text { insertion }\end{array}$ & N/A & N/A \\
\hline $\begin{array}{l}\text { Jiang et al., } \\
2014\end{array}$ & $\begin{array}{l}\text { PET group }(n=24) ; \text { PET }+ \\
\text { SF group (SF-coating group) } \\
(n=24) ; \text { PET }+ \text { SF }+ \text { HAP } \\
\text { group (combined HAP- and } \\
\text { SF-coating group) }(n=24)\end{array}$ & 4,8 weeks & N/A & $\begin{array}{l}\text { New bone tissue formation was only found } \\
\text { in the PET + SF + HAP group, the PET } \\
\text { fibers were almost completely encircled by } \\
\text { collagen, and the width of the graft-bone } \\
\text { interface was narrower than that in the } \\
\text { other two groups }\end{array}$ & $\begin{array}{l}\text { The mean load to failure and the } \\
\text { stiffness value of the PET }+ \text { SF }+ \\
\text { HAP group was higher than } \\
\text { those of the PET group and the } \\
\text { PET + SF group }\end{array}$ & $\begin{array}{l}\text { The mRNA level of BMP- } 7 \text { in the } \\
\text { PET + SF + HAP groups was } \\
\text { significantly higher than those in } \\
\text { the other two groups }\end{array}$ \\
\hline Zhao et al., 2014 & $\begin{array}{l}\text { Transosseous repair }(n= \\
\text { 48); PLGA membranes ( } n= \\
\text { 48); bFGF-PLGA } \\
\text { membranes }(n=48)\end{array}$ & $\begin{array}{l}2,4,8 \\
\text { weeks }\end{array}$ & None & $\begin{array}{l}\text { Electrospun fibrous membranes have } \\
\text { excellent biocompatibility and } \\
\text { biodegradability, and significantly } \\
\text { increased the area of glycosaminoglycan } \\
\text { staining at the tendon-bone interface } \\
\text { compared with the control group, and } \\
\text { bFGF-PLGA significantly improved } \\
\text { collagen organization }\end{array}$ & $\begin{array}{l}\text { The electrospun fibrous } \\
\text { membrane groups had a greater } \\
\text { ultimate load-to-failure and } \\
\text { stiffness than the control group, } \\
\text { and the bFGF-PLGA } \\
\text { membranes had the highest } \\
\text { ultimate load-to-failure, stiffness, } \\
\text { and stress of the healing enthesis }\end{array}$ & N/A \\
\hline $\begin{array}{l}\text { Loeffler et al., } \\
2013\end{array}$ & $\begin{array}{l}\text { Group I (control without } \\
\text { surgery); Group II (surgical } \\
\text { defect in the rotator cuff } \\
\text { only); Group III (surgical } \\
\text { defect with suture repair } \\
\text { only); Group IV (surgical } \\
\text { defect and repair with } \\
\text { sponge only); Group V } \\
\text { (surgical defect and repair } \\
\text { with sponge loaded with } \\
\text { cells) }\end{array}$ & $\begin{array}{l}3,6,12 \\
\text { weeks }\end{array}$ & None & $\begin{array}{l}\text { The cellularity, inflammation, vascularity, } \\
\text { and collagen organization increased in all } \\
\text { repaired groups, and collagen organization } \\
\text { at } 12 \text { weeks in Group } V \text { increased to } \\
\text { improve healing with cells }\end{array}$ & N/A & N/A \\
\hline $\begin{array}{l}\text { Vavken et al., } \\
2012\end{array}$ & $\begin{array}{l}\text { ACL reconstruction }(n=8) ; \\
\text { Enhanced ACL repair }(n= \\
\text { 8); ACL transection }(n=8) ;\end{array}$ & 15 weeks & None & $\mathrm{N} / \mathrm{A}$ & $\begin{array}{l}\text { There were no significant } \\
\text { differences between } \\
\text { bioenhanced ACL repair and } \\
\text { ACL reconstruction. }\end{array}$ & N/A \\
\hline Li et al., 2012 & Control group; LBL group & 4,8 weeks & N/A & $\begin{array}{l}\text { New bone formed at the graft-bone } \\
\text { interface in the LBL group, and the newly } \\
\text { formed bone was similar with the host } \\
\text { bone, and the interface between graft and } \\
\text { host bone became narrow }\end{array}$ & $\begin{array}{l}\text { The mean load-to-failure and } \\
\text { mean stiffness for the } L B L \text { group } \\
\text { was higher than that of the } \\
\text { control group at } 8 \text { weeks }\end{array}$ & N/A \\
\hline $\begin{array}{l}\text { Zhang et al., } \\
2011\end{array}$ & $\begin{array}{l}\text { Control group }(n=17) ; \\
\text { Fibrin glue-BMP group }(n= \\
\text { 17); RBX (recombined bone } \\
\text { xenograft) group }(n=17)\end{array}$ & $\begin{array}{l}2,6,12 \\
\text { weeks }\end{array}$ & N/A & $\begin{array}{l}\text { The interface of fibrin glue-BMP developed } \\
\text { new cartilage, and the interface of RBX } \\
\text { had large areas of chondrocyte-like cells, } \\
\text { bone formation and an immature } \\
\text { neo-enthesis structure }\end{array}$ & $\begin{array}{l}\text { The ultimate load of RBX group } \\
\text { was higher than tfibrin glue-BMP } \\
\text { group and control group }\end{array}$ & $\begin{array}{l}\text { Micro-CT examination showed } \\
\text { the value of bone mineral density } \\
\text { in RBX group was significantly } \\
\text { higher compared to fibrin } \\
\text { glue-BMP group and control } \\
\text { group at } 12 \text { weeks }\end{array}$ \\
\hline
\end{tabular}


graft from 4 to 12 weeks after surgery. The ligamentization period refers to the period from the proliferation period to the end of graft remodeling, which can be as long as half a year to several years. Tabuchi et al. (2012) observed the tendon implanted in the rabbit bone tunnel for 26 weeks; the results showed that Sharpeylike fibers containing type III collagen fibers were gradually replaced by type I collagen from 12 weeks after surgery until the fibers in the graft formed a connection with Sharpey-like fibers at week 26.

\section{Biomaterials of Tendon-Bone Healing and Bone Tissue Engineering Scaffolds}

Biomaterials of tissue engineering scaffold is an important and difficult point in the research of tissue engineering. Without suitable scaffolds, the seed cells will be lost and die. Tissue engineering scaffold materials should have good biocompatibility, biodegradability, three-dimensional structure, plasticity, and equivalent mechanical strength. It also has good surface activity, which is conducive to the adhesion of seed cells and provides a good microenvironment for the growth and reproduction of cells on the surface and secretion matrix. For tendon and tissue engineering scaffold materials, the most researched at present are natural materials, synthetic materials, copolymers, and composite materials. The following are commonly used and investigated in this review.

\section{Natural Materials}

The excellent biocompatibility of natural polymers such as collagen and fibrin can significantly improve the interaction between materials and tissue cells (Doty et al., 2019). In our review, collagen is widely used and evaluated in tendon bone healing. It is the main component of extracellular matrix (ECM), which can be extracted from animal bones and fascia through digestion, hydrolysis, and other processes (Shi et al., 2019; Mohammed et al., 2020). In the process of evolution, collagen retains the original amino acid sequence, and the scaffold material made by it has non-antigenicity, good biocompatibility in vivo, and permeability (Dunshee et al., 2020). Because the tissue composition of tendons is mainly composed of collagen fibers, the large fiber bundles are arranged in parallel, and their direction is consistent with the traction force they bear. Collagen fiber has toughness and strong traction resistance; in addition, the cell adhesion signal sequence contained in it can also guide the specific identification of the scaffold material by the cells. Collagen fiber has toughness and strong traction resistance (Sharma et al., 2019; Tonndorf et al., 2020). After years of development, its preparation method has been quite mature. It has now been certified by the US Food and Drug Administration (FDA) and can be successfully used in ECM scaffolds for tissue-engineered tendons. Bellincampi et al. (1998) inoculated autogenous tendon cells into collagen scaffolds and implanted them in rabbit knee joints and subcutaneously. The complex was still visible after 8 weeks. Young et al. (1998) used autologous bone MSC to adsorb on collagen gel and planted the compound on the joint of tendon. It was found that the tendon treated with MSC was thicker than the control group, and the collagen fiber assembly, seam characteristics, and load 
performance were better than the control group. In the included studies, the collage has been used as tissue engineering scaffold for tendon bone healing in many animal models (Fleming et al., 2009; Vavken et al., 2012; Bi et al., 2015; Kovacevic et al., 2015; Lee et al., 2017; Hu et al., 2018; Learn et al., 2019; Zhu M. et al., 2019), and its efficacy can be improved by utilizing a biofabrication process known as electrocompaction. Learn et al. (2019) had used electrochemically aligned collagen (ELAC) threads woven into biotextile scaffolds as grafts to repair critical infraspinatus tendon defects in New Zealand white rabbits, and it was found that woven ELAC scaffolds was intact and mechanically competent following 3 months of implantation and biological characteristics of tendon were present in the tissue around and within the scaffolds.

Acellular scaffolds play a unique role in promoting tendon bone healing. Lu et al., 2019 found an appropriate decellularization protocols for fibrocartilage tissue and prepared a novel book-shaped acellular fibrocartilage scaffold with cell-loading capability and chondrogenic inducibility for tissue-engineered fibrocartilage and bone-tendon healing. This screened scaffold alone could induce endogenous cells to satisfactorily regenerate fibrocartilage at 16 weeks, as characterized by fibrocartilaginous ECM deposition and good interface integration and may have further broad clinical applications in promoting bone-tendon healing. Then they creatively prepared two kinds of acellular scaffolds from bone or fibrocartilage tissue, namely book-type acellular bone scaffold (BABS) and book-type acellular fibrocartilage scaffold (BAFS). Histologically, both scaffolds well preserved the natural ECM structure without cellular components. In vitro studies have shown that BABS has a good ability of osteogenic induction, while BAFS has a good ability of cartilage induction. Using a rabbit partial patellectomy model, both BAFS and BABS can promote tendon-bone healing, while the BAFS was more conductive in tendon-bone healing than the BABS. This study may provide a valuable reference for screening the optimal tissue-engineering scaffold applied in tendon-bone healing ( $\mathrm{Lu}$ et al., 2019).

\section{Synthetic Materials}

Synthetic materials like PLA, polyglycolic acid (PGA) possess three main structural forms (fiber scaffold, porous foam, tubular structure). The degradation products of PLA and PGA are lactic acid and glycolic acid, respectively, which are intermediate metabolic products of the tricarboxylic acid cycle. They have good biodegradability and compatibility and will not cause inflammation, immune reactions, and cytotoxic reactions. The most widely used biodegradable biomaterials have been widely used in tissue engineering such as bone, cartilage, blood vessels, nerves, and skin (Learn et al., 2019). Cao et al. (2002) inoculated tendon cells obtained from the tendon tissues of calf shoulders and knees on a cable-like PGA mesh scaffold and implanted them subcutaneously in nude mice after 1 week in vitro culture. It was found that at 12 weeks, tendon tissue similar to the normal tendon structure was formed, and it had a certain degree of biomechanical properties. Cao et al. (2002) also used the method of autologous tendon cells + PGA + biofilm wrapping to repair the $4 \mathrm{~cm}$ tendon defect in Leghorn muscles. The results showed that the implanted tissue-engineered tendon was small, similar to the normal tendon only in general morphology and histology, and its biomechanical properties also reached $83 \%$ of the normal tendon.

Moreover, PCL, as a synthetic material, can also be used as scaffold to repair the defect of tendon bone, and many studies also have proved the application of PCL (Han et al., 2015; Zhao et al., 2015b; Han F. et al., 2019; Li et al., 2019). PCL is a kind of thermoplastic crystalline polyester obtained by ring-opening polymerization of caprolactone with diol as initiator, containing many methyl groups, so it has hydrophobicity. In addition, its degradation rate is lower than other aliphatic polyesters (Hagg et al., 1991; Crair et al., 1998). Han F. et al. (2019) have found that PCL scaffold can be loaded with biological interventions such as BMP-2 and SDF- $1 \alpha$ to form fibrous scar tissue and new bone for tendon bone healing. On other hand, the use of PET is also investigated in previous studies ( $\mathrm{Li}$ et al., 2012; Jiang et al., 2014). PET is a crystalline-saturated polyester with excellent physical and mechanical properties in a wide temperature range. Earlier, PET was mainly used in the preparation of LARS artificial ligament. Because of its poor hydrophilicity and lack of bone conductivity, it is not conducive to the growth of autogenous bone tissue, thus affecting the tendon-bone healing of the tendon in the bone tunnel and inducing ligament loosening which causes operation failure in the middle and long term after operation (Thian et al., 2006). At the same time, fibroblasts and synoviocytes cannot grow on the surface of ligament joint cavity and wrap LARS ligament to induce autologous tissue growth as the poor cytocompatibility of PET material, which will eventually lead to wear and fatigue fracture, and the shedding of wear particles will cause serious synovitis of knee joint. To solve this problem, Jiang et al. (2014) combined silk fibroin (SF) and hydroxyapatite (HAp/HA) coating by biomimetic route on the surface of PET artificial ligament; the results showed that this combination could induce graft osseointegration in the bone tunnel. Moreover, Li et al. (2012) had proven that surface coating with an organic layer-by-layer self-assembled template of chitosan and hyaluronic acid on a PET artificial ligament could be designed to promote and enhance the graft-to-bone healing after artificial ligament implantation in a bone tunnel.

\section{Copolymer}

The PLA and PGA copolymers include PLGA, poly(D-lactic acid) (PDLA), poly (L-lactic acid) (PLLA), poly(D,L-lactic acid) (PDLLA) (Kikuchi et al., 2018). Since PLGA has excellent biocompatibility and tunable mechanical and degradation properties, it is the most frequently used applications of PLA and PGA in tendon tissue engineering. PLGA not only has good biocompatibility but can also induce the upregulation of certain genes (Fujimaki et al., 2020). The degradation rate can also be controlled by changing the ratio of PLA to PGA and combining the high degradation rate of PGA and the high strength of PLA. Therefore, PLGA can also be used as a cell scaffold for artificial tendons. Fujimaki et al. (2020) and Lim et al. (2019) used PLA and PGA compound PLGA as a stent and implanted a $10-\mathrm{mm}$ defect into the autogenous tendon. The control group 
was simply replanted with PLGA. Examination after 4 weeks revealed that the cell content was significantly reduced compared with that at 2 weeks, and collagen fibers I and III were formed, and the experimental group was more obvious than the control group. The material was basically degraded at 8 weeks, and the defect was repaired well at 12 weeks, with no lymphocyte infiltration. The biomechanical strength of the experimental group was significantly higher than that of the control group, close to normal tendons. Cai et al. (2018) prepared a dual-layer aligned-random nanofibrous scaffolds SF/P(LLA-CL) using SFblended poly(L-lactic acid-co-e-caprolactone) (PLLA-CL), which could effectively augment the tendon-to-bone integration and improve gradient microstructure in a rabbit extra-articular model by inducing the new bone formation, increasing the area of fibrocartilage, and improving collagen organization and maturation.

\section{Composite Materials}

Natural materials such as collagen have good biocompatibility, but there are defects such as poor mechanical properties, very fast degradation, and poor processing performance. Synthetic materials such as high-molecular materials have defects such as low degradation rate, inflammation caused by acidic degradation products, and small mechanical properties. Thus, it is difficult for a single type of material to meet the requirements of extracellular matrix materials for tendon-bone healing. These problems can be solved by the principles and methods of composite materials. That is to say, two or more kinds of biomaterials with complementary characteristics are combined in a certain proportion and manner in order to construct a new composite material that can meet the requirements. Researchers combined several single materials through appropriate methods, comprehensively considered the advantages and disadvantages of organic materials and inorganic materials, and complemented each other to form organic/inorganic composite materials, which achieved good results in practical applications. Devin et al. (1996) composed HAp and PLA/PGA copolymer (50:50) into a porous composite matrix material and found that the compressive elastic modulus of the composite increases with the HAp composition. Therefore, the introduction of calcium-phosphorus ceramics into the PLA/PGA copolymer can improve the shortcomings of poor mechanical properties, fast degradation rate, and weak bone binding ability of the PLA/PGA copolymer. Besides, research by Serre et al. (1998) confirmed that porous materials composed of type I collagen, chondroitin sulfate, and HAp/HA can promote the attachment and growth of osteoblasts and promote the calcification of their secreted matrix. Li et al. (2017) developed a dual-layer organic/inorganic flexible bipolar fibrous membrane (nHA-PLLA) to repair the defect of upraspinatus tendon in New Zealand rabbit, which could act as a bridge between the repaired tendon and footprint, affecting the healing process in multiple ways. In other studies, Chung et al. (2017) successfully fabricated a biodegradable and synthetic tri-component graft consisting of porous poly(1,8-octanediol-co-citric acid)hydroxyapatite nanocomposites (POC-HA) and poly(l-lactide) (PLL) braids, Han et al. (2015) developed a biomimetic nanofiber membrane of polycaprolactone/nanohydroxyapatite/collagen
(PCL/nHAp/Col), and Li et al. (2014) prepared a silk-TCP-PEEK scaffold of silk, TCP, and polyether ether ketone (PEEK) to improve the healing and regeneration process of the tendonbone defect. Zhang et al. (2018) found that trichostatin A (TSA) incorporated aligned fibers of PLLA had an additive effect in directing tenogenic differentiation and confirmed that composite scaffold promoted the structural and mechanical properties of the regenerated Achilles tendon, which provides fresh insights into the regulation of tendon differentiation and a clinically applicable therapeutic approach for tendon regeneration.

\section{Bionic Scaffold}

Bionic scaffolds have been of knee interest in tissue engineering which are sufficient for tissue regeneration. Liu et al. (2017) developed a bionic random-aligned-random-tendon ECM composite scaffold for reconstruction of the soft tissue-bone junction in rabbit model. Microcomputed tomography (microCT) and histological analysis showed that the bionic scaffold enhanced tendon bone healing and fibrocartilage formation. These results demonstrated that the bionic scaffold could be a promising scaffold for ligament/tendon-bone junction repair. Lipner et al. (2015) used bionic scaffolds implanted with pluripotent cells to promote tendon-to-bone healing by promoting collagen deposition and bone formation. Adiposederived stromal cells were implanted into the repair site of rat rotator cuff model to construct nanofiber polylactic acidglycolic acid copolymer scaffolds with different mineral content gradients. Histologically, the healing interface of all groups was mainly fibrovascular scar reaction. The results showed that the tendon-to-bone healing was dominated by scar formation, which prevented any positive effect of the implanted biomimetic scaffold. In a word, the role of biomimetic scaffold in promoting tendon bone healing is not very clear, and more studies are needed to prove its positive effect.

\section{Preparation Method of Scaffolds}

Porous and three-dimensional (3D) scaffolds have been extensively used as biomaterials in the field of tissue engineering in vitro study of cell-scaffold interactions. There are many methods for preparing porous materials, such as particle filtration method, melt molding method, emulsion freeze-drying method, high-pressure gas expansion method, fiber threedimensional interweaving method, phase separation method, etc. (Freedman and Mooney, 2019). Some scholars try to use two or more methods together, or improve existing methods, optimize process parameters, in order to obtain a better pore structure. Harris et al. (1998) used the gas-foaming method combined with the particle filtration method to prepare the stent material with open large pores, which effectively overcome the disadvantages of closed pores. Mikos et al. (1993) applied lamination technology to prepare PLA and PGA into a threedimensional polymer foam with a certain shape. The micropores of the laminated layers communicate with each other to form a continuous cell structure. These are conducive to cell growth, survival, and proliferation. An intelligent processing technology called rapid prototyping has achieved rapid development in this field (Szlazak et al., 2019). The technology is based on the 
principles of dispersion and stacking. The three-dimensional model is constructed by performing layer processing on the images obtained by CT or magnetic resonance scanning of the human body, and then layered and sliced. Finally, the STL format file is transferred to the rapid prototyping machine for processing, and the various cross-sectional contours are formed by means of hot melting and cutting, and gradually stacked into a three-dimensional part. This method can be tailored according to the requirements of different patients and has the characteristics of being fast and flexible, so it has great advantages in the processing of tissue engineering scaffolds (Duty et al., 2007; Ravichandran et al., 2017).

\section{Biodegradation}

In the past 10 years, the rapid degradation of various materials in the human body is still a major problem hindering its clinical application. Because the interface of tendon bone connection involves two different biological structures, the ideal biomaterial used for tendon bone healing should have a higher standard. In other words, it is necessary to ensure that the stress at the bone-tissue interface can be reduced while matching with the degradation rate during bone healing period, and the enzymatic hydrolysis in vivo at the end of the tendon can be resisted while the degradation products will not cause changes of the internal environment $\mathrm{pH}$. Therefore, the widely developed biphasic and triphasic stents are very competitive (Atesok et al., 2016; Tang et al., 2020; Zhou et al., 2021). The degradation rate could be adjusted by ultrasonic pretreatment, and the electrostatic attraction or physical cross-linking between the molecules could be used to improve the stability of the materials and add crosslinking agents to make the internal molecular chains of the composites produce stronger cross-linking.

\section{CONCLUSION}

Functional fibrocartilage regeneration is a choke point in tendon bone healing, and the currently available tissue-engineering strategies for fibrocartilage regeneration are insufficient because of a lack of appropriate scaffold that can load large seeding cells and induce chondrogenesis of stem cells. Numerous strategies have been employed to improve tendon bone junction healing, including delivery of stem cells, bioactive factors, and synthetic materials, but these are often inadequate at recapitulating the complex structure-function relationships at native tissue interfaces Based on the results of several studies, it is found that the challenges that may be faced in the clinical process of tendon bone healing include the following: the regeneration strategy may be overwhelmed by natural scar-mediated responses, BMP2 is not an effective growth factor to promote tendon bone healing, and scaffold materials may have a negative effect on tendon bone healing.

\section{REFERENCES}

Ando, J., Sakuraba, M., Sugawara, A., Goto, A., Azuma, S., Mitsuhashi, N., et al. (2019). Free flap reconstruction of Achilles tendon and overlying skin defect
At present, except for the periosteum which has been used clinically and the curative effect is clear, the remaining methods to promote tendon-bone healing have no definite conclusions, and further research is needed to provide a more stable healing effect after ligament or tendon repair and reconstruction. Although a number of tissue engineering scaffolds have been developed and investigated, whose efficacy and safety has been proved and can be enhanced by biological interventions in this review, the researches are mainly focused on animal models which are with limitations in clinical application. On the other hand, tendon tissue engineering has high requirements for scaffold materials, and the development and research of composite materials will continue to be a hotspot for future research. Therefore, substantial clinical trials remain to be done, and continued progress in overcoming current tissue engineering challenges should allow for successful clinical practice, which is also one of the main directions of tissue engineering material research and development in the future.

\section{DATA AVAILABILITY STATEMENT}

The original contributions presented in the study are included in the article/supplementary material, further inquiries can be directed to the corresponding authors.

\section{AUTHOR CONTRIBUTIONS}

ZM, BF, and XW: substantial contributions to the conception or design of the work, or the acquisition, analysis, or interpretation of data for the work. XH, JG, ZS, BX, MY, and ZC: drafting the work or revising it critically for important intellectual content. DJ and JY: final approval of the version to be published, agreed to be accountable for all aspects of the work in ensuring that questions related to the accuracy or integrity of any part of the work are appropriately investigated and resolved.

\section{FUNDING}

This work was supported by Peking University Health Science Center (Nos. A63498-24 and BMU2018 MX023), by Peking University Third Hospital (Nos. BYSY2018004 and BMU2019GJJXK012), and by the National Key R\&D Program of China (No. 2017YFB1303000).

\section{ACKNOWLEDGMENTS}

We would like to thank Dr. Xing Wang of the Institute of Chemistry, Chinese Academy of Sciences for his suggestions and technical support.

using ALT and TFL fabricated chimeric flap. Case Rep. Plast. Surg. Hand Surg. 6, 82-85. doi: 10.1080/23320885.2019.1635023

Atesok, K., Doral, M. N., Karlsson, J., Egol, K. A., Jazrawi, L. M., Coelho, P. G., et al. (2016). Multilayer scaffolds in orthopaedic tissue engineering. Knee 
Surg. Sports Traumatol. Arthrosc. 24, 2365-2373. doi: 10.1007/s00167-014-3 453-z

Bellincampi, L. D., Closkey, R. F., Prasad, R., Zawadsky, J. P., and Dunn, M. G. (1998). Viability of fibroblast-seeded ligament analogs after autogenous implantation. J. Orthop. Res. 16, 414-420. doi: 10.1002/jor.1100160404

Bi, F., Shi, Z., Liu, A., Guo, P., and Yan, S. (2015). Anterior cruciate ligament reconstruction in a rabbit model using silk-collagen scaffold and comparison with autograft. PLoS ONE 10:e0125900. doi: 10.1371/journal.pone.0125900

Cai, J., Wang, J., Ye, K., Li, D., Ai, C., Sheng, D., et al. (2018). Dual-layer aligned-random nanofibrous scaffolds for improving gradient microstructure of tendon-to-bone healing in a rabbit extra-articular model. Int. J. Nanomed. 13, 3481-3492. doi: 10.2147/IJN.S165633

Cao, Y., Liu, Y., Liu, W., Shan, Q., Buonocore, S. D., and Cui, L. (2002). Bridging tendon defects using autologous tenocyte engineered tendon in a hen model. Plast. Reconstr. Surg. 110, 1280-1289. doi: 10.1097/01.PRS.0000025290.49889.4D

Chen, C., Liu, F., Tang, Y. F., Qu, J., Cao, Y., Zheng, C., et al. (2019). Book-shaped acellular fibrocartilage scaffold with cell-loading capability and chondrogenic inducibility for tissue-engineered fibrocartilage and bone-tendon healing. ACS Appl. Mater. Interfaces 11, 2891-2907. doi: 10.1021/acsami.8b20563

Chen, P., Cui, L., Chen, G., You, T., Li, W., Zuo, J., et al. (2019). The application of BMP-12-overexpressing mesenchymal stem cells loaded 3D-printed PLGA scaffolds in rabbit rotator cuff repair. Int. J. Biol. Macromol. 138, 79-88. doi: 10.1016/j.ijbiomac.2019.07.041

Chou, Y. C., Yeh, W. L., Chao, C. L., Hsu, Y. H., Yu, Y. H., Chen, J. K., et al. (2016). Enhancement of tendon-bone healing via the combination of biodegradable collagen-loaded nanofibrous membranes and a three-dimensional printed bone-anchoring bolt. Int. J. Nanomed. 11, 4173-4186. doi: 10.2147/IJN.S108939

Chung, E. J., Sugimoto, M. J., Koh, J. L., and Ameer, G. A. (2017). A biodegradable tri-component graft for anterior cruciate ligament reconstruction. J. Tissue Eng. Regen. Med. 11, 704-712. doi: 10.1002/term.1966

Crair, M. C., Gillespie, D. C., and Stryker, M. P. (1998). The role of visual experience in the development of columns in cat visual cortex. Science 279, 566-570. doi: $10.1126 /$ science. 279.5350 .566

Devin, J. E., Attawia, M. A., and Laurencin, C. T. (1996). Three-dimensional degradable porous polymer-ceramic matrices for use in bone repair. J. Biomater. Sci. Polym. Ed. 7, 661-669. doi: 10.1163/156856296X00435

Doty, J., Phisitkul, P., Xiao, W., Cooper, M. T., and Brigido, S. A. (2019). Update on acute achilles tendon injuries. Foot Ankle Spec. 12, 278-280. doi: $10.1177 / 1938640019856804$

Dunshee, L. C., Sullivan, M. O., and Kiick, K. L. (2020). Manipulation of the dually thermoresponsive behavior of peptide-based vesicles through modification of collagen-like peptide domains. Bioeng. Transl. Med. 5:e10145. doi: 10.1002/btm2.10145

Duty, A. O., Oest, M. E., and Guldberg, R. E. (2007). Cyclic mechanical compression increases mineralization of cell-seeded polymer scaffolds in vivo. J. Biomech. Eng. 129, 531-539. doi: 10.1115/1.2746375

Fleming, B. C., Spindler, K. P., Palmer, M. P., Magarian, E. M., and Murray, M. M. (2009). Collagen-platelet composites improve the biomechanical properties of healing anterior cruciate ligament grafts in a porcine model. Am. J. Sports Med. 37, 1554-1563. doi: 10.1177/0363546509332257

Freedman, B. R., and Mooney, D. J. (2019). Biomaterials to mimic and heal connective tissues. Adv. Mater. 31:e1806695. doi: 10.1002/adma.201806695

Fujimaki, H., Uchida, K., Inoue, G., Matsushita, O., Nemoto, N., Miyagi, M., et al. (2020). Polyglycolic acid-collagen tube combined with collagen-binding basic fibroblast growth factor accelerates gait recovery in a rat sciatic nerve critical-size defect model. J. Biomed. Mater. Res. B Appl. Biomater. 108, 326-332. doi: 10.1002/jbm.b.34391

Hagg, T., Gulati, A. K., Behzadian, M. A., Vahlsing, H. L., Varon, S., and Manthorpe, M. (1991). Nerve growth-factor promotes Cns cholinergic axonal regeneration into acellular peripheral-nerve grafts. Exp. Neurol. 112, 79-88. doi: 10.1016/0014-4886(91)90116-T

Han, F., Zhang, P., Chen, T., Lin, C., Wen, X., and Zhao, P. (2019). A LbL-assembled bioactive coating modified nanofibrous membrane for rapid tendon-bone healing in ACL reconstruction. Int. J. Nanomed. 14, 9159-9172. doi: 10.2147/IJN.S214359

Han, F., Zhang, P., Sun, Y., Lin, C., Zhao, P., and Chen, J. (2015). Hydroxyapatitedoped polycaprolactone nanofiber membrane improves tendon-bone interface healing for anterior cruciate ligament reconstruction. Int. J. Nanomed. 10, 7333-7343. doi: 10.2147/IJN.S92099

Han, L., Fang, W. L., Jin, B., Xu, S. C., Zheng, X., and Hu, Y. G. (2019). Enhancement of tendon-bone healing after rotator cuff injuries using combined therapy with mesenchymal stem cells and platelet rich plasma. Eur. Rev. Med. Pharmacol. Sci. 23, 9075-9084. doi: 10.26355/eurrev_201910_19310

Harris, L. D., Kim, B. S., and Mooney, D. J. (1998). Open pore biodegradable matrices formed with gas foaming. J. Biomed. Mater. Res. 42, 396-402. doi: 10. 1002/(SICI)1097-4636(19981205)42:3<396::AID-JBM7>3.0.CO;2-E

Hayashida, K., Saijo, H., and Fujioka, M. (2018). Peroneal perforator-based peroneus longus tendon and sural neurofasciocutaneous composite flap transfer for a large soft-tissue defect of the forearm: a case report. Microsurgery 38, 85-88. doi: 10.1002/micr.30104

Hejbol, E. K., Sellathurai, J., Nair, P. D., and Schroder, H. D. (2017). Injectable scaffold materials differ in their cell instructive effects on primary human myoblasts. J. Tissue Eng. 8:2041731417717677. doi: 10.1177/2041731417717677

Hu, Y., Ran, J., Zheng, Z., Jin, Z., Chen, X., Yin, Z., et al. (2018). Exogenous stromal derived factor-1 releasing silk scaffold combined with intra-articular injection of progenitor cells promotes bone-ligament-bone regeneration. Acta Biomater. 71, 168-183. doi: 10.1016/j.actbio.2018.02.019

Huang, Y., Pan, M., Shu, H., He, B., Zhang, F., and Sun, L. (2020). Vascular endothelial growth factor enhances tendon-bone healing by activating Yesassociated protein for angiogenesis induction and rotator cuff reconstruction in rats. J. Cell Biochem. 121, 2343-2353. doi: 10.1002/jcb.29457

Iwahashi, T., Shino, K., Nakata, K., Otsubo, H., Suzuki, T., Amano, H., et al. (2010). Direct anterior cruciate ligament insertion to the femur assessed by histology and 3-dimensional volume-rendered computed tomography. Arthroscopy 26, S13-20. doi: 10.1016/j.arthro.2010.01.023

Jiang, J., Wan, F., Yang, J., Hao, W., Wang, Y., Yao, J., et al. (2014). Enhancement of osseointegration of polyethylene terephthalate artificial ligament by coating of silk fibroin and depositing of hydroxyapatite. Int. J. Nanomed. 9, 4569-4580. doi: $10.2147 /$ IJN.S69137

Karel, S., Sogorkova, J., Hermannova, M., Nesporova, K., Marholdova, L., Chmelickova, K., et al. (2018). Stabilization of hyaluronan-based materials by peptide conjugation and its use as a cell-seeded scaffold in tissue engineering. Carbohydr. Polym. 201, 300-307. doi: 10.1016/j.carbpol.2018.08.082

Khoo, R., and Nikkhah, D. (2019). Flexor tendon retrieval of multidigit flexor zone 2 injuries using push-pull technique: video and technical considerations. Plast. Reconstr. Surg. 144, 1129e-1130e. doi: 10.1097/PRS.0000000000006285

Kikuchi, D., Iizuka, T., Nomura, K., Kuribayashi, Y., Tanaka, M., Yamashita, S., et al. (2018). Feasibility of autologous fibrin glue and polyglycolic acid sheets to prevent delayed bleeding after endoscopic submucosal dissection of gastric neoplasms in patients receiving antithrombotic therapy. Gastroenterol. Res. Pract. 2018:2174957. doi: 10.1155/2018/2174957

Kovacevic, D., Gulotta, L. V., Ying, L., Ehteshami, J. R., Deng, X. H., and Rodeo, S. A. (2015). rhPDGF-BB promotes early healing in a rat rotator cuff repair model. Clin. Orthop. Relat. Res. 473, 1644-1654. doi: 10.1007/s11999-0144020-0

Kunze, K. N., Burnett, R. A., Shinsako, K. K., Bush-Joseph, C. A., Cole, B. J., and Chahla, J. (2019). Trapezoidal achilles tendon allograft plug for revision quadriceps tendon repair with a large tendon defect. Arthrosc. Tech. 8, e1031e1036. doi: 10.1016/j.eats.2019.05.015

Learn, G. D., Mcclellan, P. E., Knapik, D. M., Cumsky, J. L., Webster-Wood, V., Anderson, J. M., et al. (2019). Woven collagen biotextiles enable mechanically functional rotator cuff tendon regeneration during repair of segmental tendon defects in vivo. J. Biomed. Mater. Res. B Appl. Biomater. 107, 1864-1876. doi: 10.1002/jbm.b.34279

Lee, K. W., Lee, J. S., Jang, J. W., Shim, Y. B., and Lee, K. I. (2017). Tendonbone interface healing using an injectable rhBMP-2-containing collagen gel in a rabbit extra-articular bone tunnel model. J. Tissue Eng. Regen. Med. 11, 1435-1441. doi: 10.1002/term.2041

Lewandowska-Lancucka, J., Fiejdasz, S., Rodzik, L., Koziel, M., and Nowakowska, M. (2015). Bioactive hydrogel-nanosilica hybrid materials: a potential injectable scaffold for bone tissue engineering. Biomed. Mater. 10:015020. doi: 10.1088/1748-6041/10/1/015020

Li, H., Chen, Y., and Chen, S. (2019). Enhancement of rotator cuff tendon-bone healing using bone marrow-stimulating technique along with hyaluronic acid. J. Orthop. Translat. 17, 96-102. doi: 10.1016/j.jot.2019.01.001 
Li, H., Ge, Y., Zhang, P., Wu, L., and Chen, S. (2012). The effect of layer-by-layer chitosan-hyaluronic acid coating on graft-to-bone healing of a poly(ethylene terephthalate) artificial ligament. J. Biomater. Sci. Polym. Ed. 23, 425-438. doi: 10.1163/092050610X551989

Li, X., Cheng, R., Sun, Z., Su, W., Pan, G., Zhao, S., et al. (2017). Flexible bipolar nanofibrous membranes for improving gradient microstructure in tendon-tobone healing. Acta Biomater. 61, 204-216. doi: 10.1016/j.actbio.2017.07.044

Li, X., He, J., Bian, W., Li, Z., Li, D., and Snedeker, J. G. (2014). A novel silkTCP-PEEK construct for anterior cruciate ligament reconstruction: an offthe shelf alternative to a bone-tendon-bone autograft. Biofabrication 6:015010. doi: 10.1088/1758-5082/6/1/015010

Lim, W. L., Liau, L. L., Ng, M. H., Chowdhury, S. R., and Law, J. X. (2019). Current progress in tendon and ligament tissue engineering. Tissue Eng. Regen. Med. 16, 549-571. doi: 10.1007/s13770-019-00196-w

Lipner, J., Shen, H., Cavinatto, L., Liu, W. Y., Havlioglu, N., Xia, Y. N., et al. (2015). In vivo evaluation of adipose-derived stromal cells delivered with a nanofiber scaffold for tendon-to-bone repair. Tissue Eng. Part A 21, 2766-2774. doi: 10.1089/ten.tea.2015.0101

Liu, H. H., Yang, L., Zhang, E. C., Zhang, R., Cai, D. D., Zhu, S. A., et al. (2017). Biomimetic tendon extracellular matrix composite gradient scaffold enhances ligament-to-bone junction reconstruction. Acta Biomater. 56, 129-140. doi: 10.1016/j.actbio.2017.05.027

Loeffler, B. J., Scannell, B. P., Peindl, R. D., Connor, P., Davis, D. E., Hoelscher, G. L., et al. (2013). Cell-based tissue engineering augments tendon-to-bone healing in a rat supraspinatus model. J. Orthop. Res. 31, 407-412. doi: 10.1002/jor.22234

Lu, H. B., Tang, Y. F., Liu, F., Xie, S. S., Qu, J., and Chen, C. (2019). Comparative evaluation of the book-type acellular bone scaffold and fibrocartilage scaffold for bone-tendon healing. J. Orthop. Res. 37, 1709-1722. doi: 10.1002/jor.24301

Maher, C. G., Sherrington, C., Herbert, R. D., Moseley, A. M., and Elkins, M. (2003). Reliability of the PEDro scale for rating quality of randomized controlled trials. Phys. Ther. 83, 713-721. doi: 10.1093/ptj/83.8.713

Mikos, A. G., Sarakinos, G., Leite, S. M., Vacanti, J. P., and Langer, R. (1993). Laminated three-dimensional biodegradable foams for use in tissue engineering. Biomaterials 14, 323-330. doi: 10.1016/0142-9612(93)90049-8

Mochizuki, T., Fujishiro, H., Nimura, A., Mahakkanukrauh, P., Yasuda, K., Muneta, T., et al. (2014). Anatomic and histologic analysis of the mid-substance and fan-like extension fibres of the anterior cruciate ligament during knee motion, with special reference to the femoral attachment. Knee Surg. Sports Traumatol. Arthrosc. 22, 336-344. doi: 10.1007/s00167-013-2404-4

Mochizuki, T., Muneta, T., Nagase, T., Shirasawa, S., Akita, K. I., and Sekiya, I. (2006). Cadaveric knee observation study for describing anatomic femoral tunnel placement for two-bundle anterior cruciate ligament reconstruction. Arthroscopy 22, 356-361. doi: 10.1016/j.arthro.2005.09.020

Mochizuki, Y., and Ochi, M. (2015). Clinical results of arthroscopic polyglycolic acid sheet patch graft for irreparable rotator cuff tears. Asia Pac. J. Sports Med. Arthrosc. Rehabil. Technol. 2, 31-35. doi: 10.1016/j.asmart.2014.11.002

Mohammed, D., Pardon, G., Versaevel, M., Bruyere, C., Alaimo, L., Luciano, M., et al. (2020). Producing collagen micro-stripes with aligned fibers for cell migration assays. Cell Mol. Bioeng. 13, 87-98. doi: 10.1007/s12195-019-00600-4

Periasamy, M., Venkatramani, H., and Shanmuganathan, R. S. (2019). Management of chronic achilles tendon injuries-review of current protocols and surgical options. Indian J. Plast Surg. 52, 109-116. doi: 10.1055/s-0039-1687923

Petruskevicius, J., Nielsen, S., Kaalund, S., Knudsen, P. R., and Overgaard, S. (2002). No effect of osteoset, a bone graft substitute, on bone healing in humans: a prospective randomized double-blind study. Acta Orthop. Scand. 73, 575-578. doi: 10.1080/000164702321022875

Ravichandran, A., Lim, J., Chong, M. S. K., Wen, F., Liu, Y., Pillay, Y. T., et al. (2017). In vitro cyclic compressive loads potentiate early osteogenic events in engineered bone tissue. J. Biomed. Mater. Res. B Appl. Biomater. 105, 2366-2375. doi: 10.1002/jbm.b.33772

Rodriguez-Vazquez, M., and Ramos-Zuniga, R. (2020). Chitosan-hydroxyapatite scaffold for tissue engineering in experimental lumbar laminectomy and posterolateral spinal fusion in wistar rats. Asian Spine J. 14, 139-147. doi: 10.31616/asj.2019.0091

Sadeghinia, A., Soltani, S., Aghazadeh, M., Khalilifard, J., and Davaran, S. (2020). Design and fabrication of clinoptilolite-nanohydroxyapatite/chitosan-gelatin composite scaffold and evaluation of its effects on bone tissue engineering. J. Biomed. Mater. Res. A 108, 221-233. doi: 10.1002/jbm.a.36806

Sasaki, N., Ishibashi, Y., Tsuda, E., Yamamoto, Y., Maeda, S., Mizukami, H., et al. (2012). The femoral insertion of the anterior cruciate ligament: discrepancy between macroscopic and histological observations. Arthroscopy 28, 1135-1146. doi: 10.1016/j.arthro.2011.12.021

Serre, C. M., Papillard, M., Chavassieux, P., Voegel, J. C., and Boivin, G. (1998). Influence of magnesium substitution on a collagen-apatite biomaterial on the production of a calcifying matrix by human osteoblasts. J. Biomed. Mater. Res. 42, 626-633. doi: 10.1002/(SICI) 1097-4636(19981215)42:4<626::AIDJBM20>3.0.CO;2-S

Sharma, V., Kumar, A., Puri, K., Bansal, M., and Khatri, M. (2019). Application of platelet-rich fibrin membrane and collagen dressing as palatal bandage for wound healing: a randomized clinical control trial. Indian J. Dent. Res. 30, 881-888. doi: 10.4103/ijdr.IJDR_370_17

Shi, Y., Helary, C., and Coradin, T. (2019). Exploring the cellprotein-mineral interfaces: interplay of silica (nano)rods@collagen biocomposites with human dermal fibroblasts. Mater. Today Bio. 1:100004. doi: 10.1016/j.mtbio.2019.100004

Szlazak, K., Vass, V., Hasslinger, P., Jaroszewicz, J., Dejaco, A., Idaszek, J., et al. (2019). X-ray physics-based CT-to-composition conversion applied to a tissue engineering scaffold, enabling multiscale simulation of its elastic behavior. Mater. Sci. Eng. C Mater. Biol. Appl. 95, 389-396. doi: 10.1016/j.msec.2017.11.044

Tabuchi, K., Soejima, T., Kanazawa, T., Noguchi, K., and Nagata, K. (2012). Chronological changes in the collagen-type composition at tendon-bone interface in rabbits. Bone Joint Res. 1, 218-224. doi: 10.1302/2046-3758.19.2000109

Tang, Y., Chen, C., Liu, F., Xie, S., Qu, J., Li, M., et al. (2020). Structure and ingredient-based biomimetic scaffolds combining with autologous bone marrow-derived mesenchymal stem cell sheets for bone-tendon healing. Biomaterials 241:119837. doi: 10.1016/j.biomaterials.2020.119837

Thian, E. S., Huang, J., Best, S. M., Barber, Z. H., Brooks, R. A., Rushton, N., et al. (2006). The response of osteoblasts to nanocrystalline siliconsubstituted hydroxyapatite thin films. Biomaterials 27, 2692-2698. doi: 10.1016/j.biomaterials.2005.12.019

Tonndorf, R., Aibibu, D., and Cherif, C. (2020). Corrigendum to "collagen multifilament spinning” [Mater. Sci. Eng. C. 106 (2020): 110105]. Mater. Sci. Eng. C Mater. Biol. Appl. 109:110513. doi: 10.1016/j.msec.2019.110513

Uz, U., Gunhan, K., Vatansever, S., Kivanc, M., and Yuceturk, A. V. (2019). Novel simple strategy for cartilage tissue engineering using stem cells and synthetic polymer scaffold. J. Craniofac. Surg. 30, 940-943. doi: 10.1097/SCS.0000000000005374

Van Der Made, A. D., Tol, J. L., Reurink, G., Peters, R. W., and Kerkhoffs, G. M. (2019). Potential hamstring injury blind spot: we need to raise awareness of proximal hamstring tendon avulsion injuries. Br. J. Sports Med. 53, 390-392. doi: 10.1136/bjsports-2018-100063

Vavken, P., Fleming, B. C., Mastrangelo, A. N., Machan, J. T., and Murray, M. M. (2012). Biomechanical outcomes after bioenhanced anterior cruciate ligament repair and anterior cruciate ligament reconstruction are equal in a porcine model. Arthroscopy 28, 672-680. doi: 10.1016/j.arthro.2011.10.008

Weiler, A., Hoffmann, R. F., Bail, H. J., Rehm, O., and Sudkamp, N. P. (2002) Tendon healing in a bone tunnel. Part II: histologic analysis after biodegradable interference fit fixation in a model of anterior cruciate ligament reconstruction in sheep. Arthroscopy 18, 124-135. doi: 10.1053/jars.2002.30657

Wisbech Vange, S., Tranum-Jensen, J., and Krogsgaard, M. R. (2020). Gracilis tendon harvest may lead to both incisional and non-incisional saphenous nerve injuries. Knee Surg. Sports Traumatol. Arthrosc. 28, 969-974. doi: 10.1007/s00167-019-05605-0

Xu, Q., Sun, W. X., and Zhang, Z. F. (2019). High expression of VEGFA in MSCs promotes tendon-bone healing of rotator cuff tear via microRNA-205-5p. Eur. Rev. Med. Pharmacol. Sci 23, 4081-4088. doi: 10.26355/eurrev_201905_17909

Young, R. G., Butler, D. L., Weber, W., Caplan, A. I., Gordon, S. L., and Fink, D. J. (1998). Use of mesenchymal stem cells in a collagen matrix for Achilles tendon repair. J. Orthop. Res. 16, 406-413. doi: 10.1002/jor.1100160403

Zhang, C., Wang, X. L., Zhang, E. C., Yang, L., Yuan, H. H., Tu, W. J., et al. (2018). An epigenetic bioactive composite scaffold with well-aligned 
nanofibers for functional tendon tissue engineering. Acta Biomater. 66, 141-156. doi: 10.1016/j.actbio.2017.09.036

Zhang, P., Han, F., Chen, T., Wu, Z., and Chen, S. (2020). "Swiss roll"-like bioactive hybrid scaffolds for promoting bone tissue ingrowth and tendonbone healing after anterior cruciate ligament reconstruction. Biomater. Sci. 8, 871-883. doi: 10.1039/C9BM01703H

Zhang, W., Pan, W., Zhang, M., and Wei, Y. (2011). In vivo evaluation of two types of bioactive scaffold used for tendon-bone interface healing in the reconstruction of anterior cruciate ligament. Biotechnol. Lett. 33, 837-844. doi: 10.1007/s10529-010-0490-7

Zhao, F., Hu, X., Zhang, J., Shi, W., Ren, B., Huang, H., et al. (2019). A more flattened bone tunnel has a positive effect on tendon-bone healing in the early period after ACL reconstruction. Knee Surg. Sports Traumatol. Arthrosc. 27, 3543-3551. doi: 10.1007/s00167-019-05420-7

Zhao, S., Xie, X., Pan, G., Shen, P., Zhao, J., and Cui, W. (2015a). Healing improvement after rotator cuff repair using gelatin-grafted poly(Llactide) electrospun fibrous membranes. J. Surg. Res. 193, 33-42. doi: $10.1016 /$ j.jss.2014.08.019

Zhao, S., Zhao, J., Dong, S., Huangfu, X., Li, B., Yang, H., et al. (2014). Biological augmentation of rotator cuff repair using bFGF-loaded electrospun poly(lactide-co-glycolide) fibrous membranes. Int. J. Nanomed. 9, 2373-2385. doi: 10.2147/IJN.S59536

Zhao, S., Zhao, X., Dong, S., Yu, J., Pan, G., Zhang, Y., et al. (2015b). A hierarchical, stretchable and stiff fibrous biotemplate engineered using staggerelectrospinning for augmentation of rotator cuff tendon-healing. J. Mater. Chem. B 3, 990-1000. doi: 10.1039/C4TB01642D
Zhou, Y., Xie, S., Tang, Y., Li, X., Cao, Y., Hu, J., et al. (2021). Effect of book-shaped acellular tendon scaffold with bone marrow mesenchymal stem cells sheets on bone-tendon interface healing. J. Orthop. Trans. 26, 162-170. doi: 10.1016/j.jot.2020. 02.013

Zhu, M., Tay, M. L., Callon, K., Tuari, D., Zhao, L., Dray, M., et al. (2019). Overlay repair with a synthetic collagen scaffold improves the quality of healing in a rat rotator cuff repair model. J. Shoulder Elbow Surg. 28, 949-958. doi: 10.1016/j.jse.2018. 11.044

Zhu, Q., Ma, Z. J., Li, H. Y., Wang, H. M., and He, Y. H. (2019). Enhancement of rotator cuff tendon-bone healing using combined aligned electrospun fibrous membranes and kartogenin. Rsc. Adv. 9, 15582-15592. doi: 10.1039/C8RA0 9849B

Conflict of Interest: The authors declare that the research was conducted in the absence of any commercial or financial relationships that could be construed as a potential conflict of interest.

Copyright (C) 2021 Mao, Fan, Wang, Huang, Guan, Sun, Xu, Yang, Chen, Jiang and $Y u$. This is an open-access article distributed under the terms of the Creative Commons Attribution License (CC BY). The use, distribution or reproduction in other forums is permitted, provided the original author(s) and the copyright owner(s) are credited and that the original publication in this journal is cited, in accordance with accepted academic practice. No use, distribution or reproduction is permitted which does not comply with these terms. 